\title{
Effect of Sediment Composition on the Uniformity of Experimentally-Formed Methane Hydrate
}

Terrence R. Ryan

West Virginia University

Follow this and additional works at: https://researchrepository.wvu.edu/etd

\section{Recommended Citation}

Ryan, Terrence R., "Effect of Sediment Composition on the Uniformity of Experimentally-Formed Methane Hydrate" (2012). Graduate Theses, Dissertations, and Problem Reports. 184.

https://researchrepository.wvu.edu/etd/184

This Thesis is protected by copyright and/or related rights. It has been brought to you by the The Research Repository @ WVU with permission from the rights-holder(s). You are free to use this Thesis in any way that is permitted by the copyright and related rights legislation that applies to your use. For other uses you must obtain permission from the rights-holder(s) directly, unless additional rights are indicated by a Creative Commons license in the record and/ or on the work itself. This Thesis has been accepted for inclusion in WVU Graduate Theses, Dissertations, and Problem Reports collection by an authorized administrator of The Research Repository @ WVU. For more information, please contact researchrepository@mail.wvu.edu. 


\title{
Effect of Sediment Composition on the Uniformity of Experimentally-Formed Methane Hydrate
}

\author{
Terrence R. Ryan
}

Thesis submitted to the College of Engineering and Mineral Resources at West Virginia University in partial fulfillment of the requirements for the degree of

\author{
Master of Science \\ in
}

Chemical Engineering

\author{
Brian Anderson, Ph.D., Chair \\ Charter Stinespring, Ph.D. \\ Kelly Rose, M.S.
}

Department of Chemical Engineering

Morgantown, West Virginia

2012

Keywords: Methane Hydrate; Sediment; Clay; Composition;

Experiment; Uniform; Natural Environment

Copyright 2012 Terrence R. Ryan 


\section{Abstract \\ Effect of Sediment Composition on the Uniformity of Experimentally-Formed Methane Hydrate}

\section{Terrence R. Ryan}

Experimentalists that try to duplicate the methane hydrate found in natural systems have regularly made non-uniform hydrate in packed sediments. Some element of the natural system is missing from the experimental procedure. The goal of this research was to discover what role sediment composition and grain size play in the uniformity of experimentally-formed methane hydrate. The discoveries were quite surprising.

The motivation for this research lies in the analysis of sediment samples and well-log data from the 2010 Mount Elbert, AK Stratigraphic Test Well. Sedimentological analysis of core samples from this well indicated that a thin section within the methane hydrate formation lacked the kaolinite clay that was found throughout the rest of test well. This section also corresponded to a void in the methane hydrate saturation. Thus, a hypothesis was developed which states that a critical amount of clay (such as kaolinite) is needed in a sediment matrix for a uniform methane hydrate formation to occur.

To test the hypothesis, a three-part experiment was performed. First, a method of manufacturing artificial sediments was developed. This produced material which was appeared very similar to natural sediments using Scanning Electron Microscopy. Secondly, in order to achieve packing characteristics similar to a natural environment, a centrifuge-based sediment packing system was constructed, and a sediment packing procedure was developed. Lastly, the packed artificial sediment was used in a methane hydrate formation experiment conducted in a Computed Tomography (CT) Scanner. Use of the CT scanner would allow imaging of the resultant methane hydrate formation.

A total of three experiments were conducted. Two experiments were conducted on sediments containing quartz grains mixed with kaolinite clay. The third experiment was conducted on sediment containing only quartz. In the two experiments involving 
kaolinite-quartz sediments, the resulting methane hydrate formation was highly uniform. The methane hydrate formed in the quartz-only sediment was highly non-uniform.

The positive effect of the kaolinite clay on the uniformity of a methane hydrate formation is therefore taken to be true. The presence of clay in sediment allows water to be distributed uniformly. If the water is distributed uniformly, the resultant methane hydrate should be uniformly distributed, as well. The water is not subject to rapid movement during the methane hydrate formation from gravity or concentration gradients.

To the knowledge of those involved in this work, this is the first time uniform methane hydrate has been formed in artificial packed sediment. Other experimentalists can apply these techniques to their own work and broaden the knowledge base of the hydrate community. This discovery may also help explain why hydrate does not form properly in what may be an otherwise perfect environment. This work is just the beginning. 


\section{Acknowledgements}

I would like to acknowledge the help of many people in the performance of the work detailed in this thesis.

First and foremost, I would like to thank my wife Cindy, for all her love, patience, and understanding as I undertook this endeavor. Without her, my graduate education would have not been possible.

Next, I would like to thank my son, Patrick, for helping me understand what it took to be a student again. Beginning in his first grade year, Patrick got to watch his dad go through the same struggle with classes and homework that he did. His encouragement through this process was a great bonding experience.

Thanks also go to the members of my Committee - Dr. Brian Anderson, Dr. Charter Stinespring, and Ms. (and future Dr.) Kelly Rose. The help and guidance from all of them allowed this work to come to fruition.

Also deserving recognition are the people working at NETL-Morgantown, WV. Dr. Yongkoo Seol graciously shared his equipment. Dr. Jeung Choi offered guidance in the operation of the methane hydrate cart. Dr. Dustin McIntyre allowed me great latitude in using the facilities at NETL. Dr. Dustin Crandall provided assistance with manipulating images with ImageJ software. Karl Jarvis and Brian Tennant were invaluable in setting up and operating the methane hydrate cart and CT scanner. 
The experience and imagination of Jim Hall in the WVU Department of Chemical Engineering Shop was crucial to manufacturing many of the devices needed to complete my experimental work.

The members of Dr. Anderson's research group and the other Chemical Engineering graduate students deserve my thanks, as well. Whether it was help with homework, class selection, presentations, or meeting the challenges of writing a thesis, the group always provided a helping hand. 


\section{Table of Contents}

1 Motivation for Experimental Study ................................................................ 1

2 Description of Mount Elbert, Alaska Methane Hydrate Formation ......................... 3

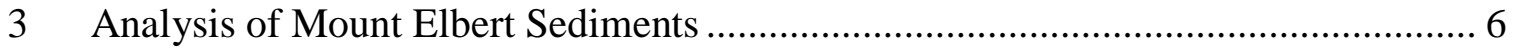

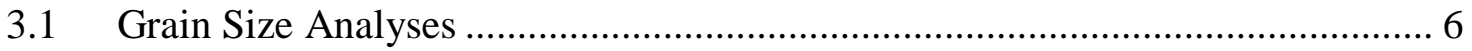

3.2 X-Ray Diffraction Analyses ................................................................. 7

3.3 Scanning Electron Microscopy Analyses .................................................. 10

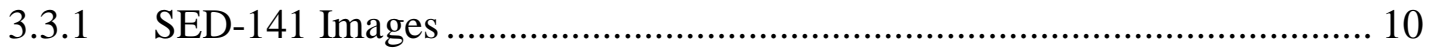

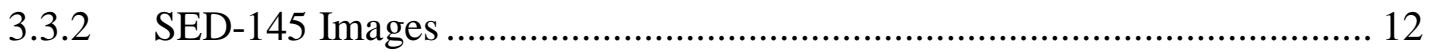

4 Development of Hypothesis Concerning Sediment Composition .......................... 15

4.1 Review of Observations concerning Mount Elbert Reservoir ......................... 15

4.2 Discussion of How Observations Lead to Hypothesis ..................................... 15

4.3 Sediment Composition Plays an Important Role in Hydrate Formation............ 16

5 Experimental References and Current Laboratory Methodology ............................. 16

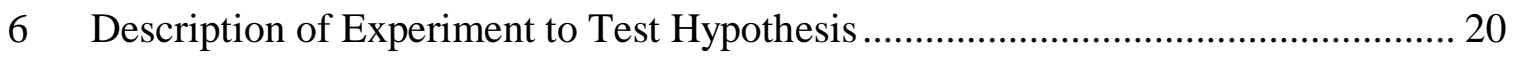

6.1 Preparation of Artificial Sediment Samples ............................................... 20

6.1.1 Preparation of Large Grain Quartz/Kaolinite Artificial Sediment.............. 21

6.1.2 Preparation of Large Grain Quartz/Ground Quartz Artificial Sediment .... 22

6.1.3 Visual Comparison of Kaolinite/Quartz versus Ground Quartz/Quartz

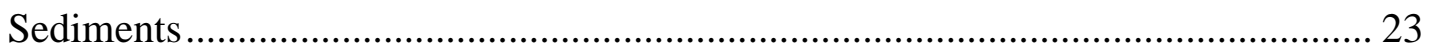

6.1.4 SEM Imaging of Prepared Sediments ................................................... 23

6.2 Sediment Packing Procedure ............................................................................. 29

6.3 Visual Sediment Packing Experiments ................................................... 34

6.4 Computed Tomography (CT) Scanning of Artificial Sediment Mixtures ......... 37

6.5 Experimental Apparatus for Methane Hydrate Formation Experiments ........... 38

6.6 Experimental Procedure to Test Primary Hypothesis .................................... 42

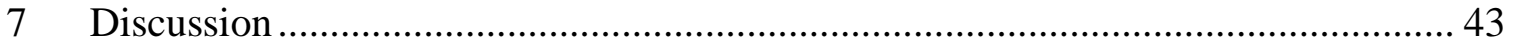

7.1 Experiment \#1: 90\% F110 Sand with 10\% kaolinite and 10 grams of water .... 44

7.2 Experiment \#2: 90\% F110 Sand with 10\% kaolinite and 20 grams of water .. 48

7.3 Experiment \#3: 90\% F110 Sand with 10\% quartz fines and 20 grams of water 52

8 Conclusions 


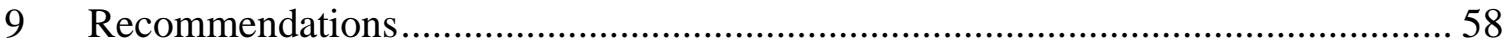

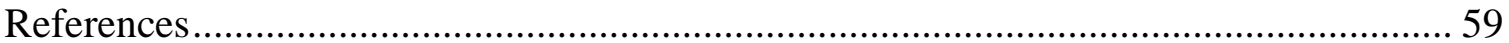

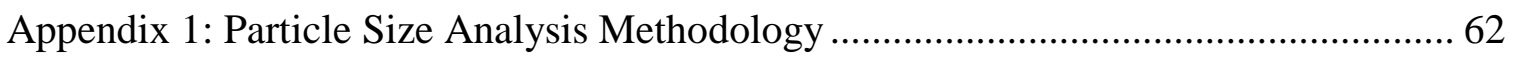

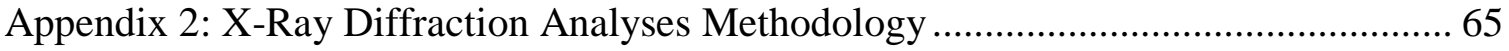

Appendix 3: SEM Sample Preparation and Imaging Procedure ..................................... 68

Appendix 4: Lithostatic Force Calculation of Sediment Packing .................................. 70 


\section{List of Figures}

Figure 1: Lithostratigraphic Montage of Mount Elbert, AK Reservoir

Figure 2: SED-141 Image 1 - Sample at 250X magnification. Note the clay coating, which gives the coated quartz grains a fuzzy appearance. Particles of clay and small quartz grains can be seen in the background on the mounting tape....

Figure 3: SED-141 Image 2 - Sample at 25,000X magnification. Individual grains of clay can be seen adhering to the quartz grain and to other clay particles.....

Figure 4: SED-145 Image 1 - Sample at 50X magnification. On several of the larger grains, flat surfaces can be seen. Random agglomeration of smaller fines can be seen throughout the image.

Figure 5: SED-145 Image 2 - Sample at 250X magnification. The fine grains do not adhere uniformly to the larger quartz grains.

Figure 6: 250X Magnification of F110 Sand Grains as received from US Silica

Figure 7: 600X magnification of quartz/kaolinite artificial sediment showing fuzzy texture, which is an indication of uniform coating of the large quartz grain.

Figure 8: 10,000X magnification of kaolinite coating on the surface of the large grain quartz, showing individual grains of kaolinite clay layered on the surface.....

Figure 9: 250X magnification of quartz fines on large grained quartz. Note the surface detail of the grain, which indicated the lack of coating of fine grain quartz

Figure 10: 2,500X magnification of quartz fines on large grain quartz. Note the sparse coating of quartz fines on the surface of the large quartz grain. The large quartz grain is virtually uncoated by the fine grain quartz.

Figure 11: 10,000X magnification of quartz fines on large grain quartz surface. The $1 \mu \mathrm{m}$ fine quartz grains tended to agglomerate in irregularities on the surface of the large quartz grains. Unlike the kaolinite, the fine quartz grains did not create a uniform coating over the surface of the large quartz grain.

Figure 12: Thermo Electron PR 7000M Centrifuge ................................................... 30

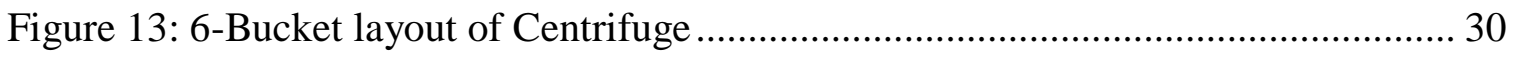

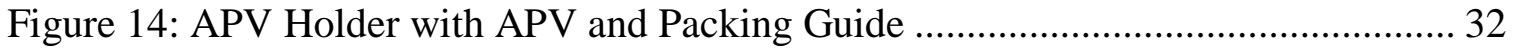

Figure 15: Packing Weight \& Guide with Rubber Base Support ............................... 33 
Figure 16: Empty polycarbonate tube with epoxy resin seal at the base

Figure 17: Wetted artificial sediments in polycarbonate tubes 37

Figure 18: Methane hydrate experimental formation apparatus P\&ID 39

Figure 19: Glycol cooling jacket in CT scanner 40

Figure 20: Glycol cooling jacket. 40

Figure 21: Interior view of glycol cooling jacket 41

Figure 22: View into top opening of glycol cooling jacket 41

Figure 23: Experiment \#1 false-color image of hydrate-wet CT scans 45

Figure 24: CT images showing hydrate saturation after the completion of hydrate formation. Circles on top of each image represent layer configuration, with open area for fine sand and filled area for coarse sand. A brighter elliptical artifact in the first three images was caused by accidental air injection during one of the later steps of the measurement sequence - from Seol \& Kneafsey ${ }^{10}$ experiment

Figure 25: Reservoir \& Exit Pressure for Experiment \#1 - The exit pressure dropped slowly from Time 0 until 6 hours into the experiment. From 6 hours until 15 hours, the exit pressure drop was more rapid. After hour 15, the exit pressure did not change....... 47

Figure 26: Glycol Bath Temperature for Experiment \#1 - The temperature showed little variation throughout the entire experiment. From Time 0 until hour 2 and from hour 16 to hour 22 , a $0.25^{\circ} \mathrm{C}$ variation is due to the heat generated by the CT scanner. Overnight, the CT scanner was turned off.

Figure 27: Reservoir \& Exit Pressure for Experiment \#2.

Figure 28: Glycol Bath and Sediment Core Temperatures for Experiment \#2.

Figure 29: Experiment \#2 False-color Image of Hydrate-Wet CT scans - most of the hydrate formed in this experiment is of relative medium density. Some irregularities can be seen, but the bulk of the hydrate is uniform.

Figure 30: Reservoir \& Exit Pressure for Experiment \#3 ......................................... 53

Figure 31: Glycol Bath and Sediment Core Temperatures for Experiment \#3................ 54

Figure 32: Experiment \#3 False-color Image of Hydrate-Wet CT Scans ........................ 55

Figure 33: Reservoir and Exit Pressures for Experiments \#2 and \#3 .............................56 


\section{$\underline{\text { List of Tables }}$}

Table 1: Grain Size via Laser Diffraction (from Rose, et al, $2011^{2}$ ) ............................ 7

Table 2: Composition via X-Ray Diffraction (from Rose, et al, $2011^{2}$ )........................ 8 


\section{$1 \quad$ Motivation for Experimental Study}

The US Department of Energy's National Energy Technology Laboratory (NETL) has a long history of research in the area of methane hydrates. This research is divided into many parts - two of which are geological field studies and methane hydrate laboratory formation studies.

The geological field studies work at NETL involves removing sediment cores from naturally occurring methane hydrate formations in both marine and terrestrial formations, and characterizing the surrounding geological environment. The intent is not to recover the methane hydrate from the formation, but to retrieve the sediment cores for further study. The sediment cores are identified, containerized, and frozen. Some field analyses and observations are conducted at the retrieval site. The core sections deemed to have the most interesting geological features are transported to laboratories for further study. These core-based observations are tied back to field-scale datasets, such as wire-line logs.

Methane hydrate laboratory formation studies are conducted at NETL laboratories, universities, and other national laboratories. Depending on the focus of the researcher, the formation experiments may attempt to interpret a particular phenomenon associated with the formation process. The NETL site in Morgantown, WV has the capability to conduct Computed Tomography (CT) scanning during methane hydrate formation experiments, which is useful in characterizing the growth of gas hydrate in sediments in situ.

During the analysis of sediment cores from the Mount Elbert site, a particular phenomenon was identified. Within a sandstone interval that was methane-rich, the 
hydrate was not present for approximately two meters within the middle of the sandstone sequence. Mineralogy analysis of the sediment cores revealed that a sample from this location was comprised of nearly pure quartz. All other samples from the formation showed the presence of kaolinite or chlorite, typically at a composition of ten percent. A hypothesis was formed which states that sediment composition plays a role in how methane hydrate forms in naturally occurring sediments.

With this data in hand, an experiment was devised that would test the theory that mineralogy plays a role in how and where methane hydrate forms in the environment. The experiment would involve conducting additional analyses of the natural sediment samples, preparing artificial sediments that mimic the natural samples, developing a method for compacting the artificial sediments, and forming hydrate in the artificial sediment.

This thesis details novel ideas for sediment preparation and compaction, which differ from traditional methods used by previous methane hydrate experimenters. The goal of the experiment was to prove the composition of sediment within which methane hydrate is formed controls the uniformity of hydrate formation within the sediment. Ultimately, it is hoped that the experiment will shed light on why methane hydrate forms in some sediments, and not in others. 


\section{Description of Mount Elbert, Alaska Methane Hydrate Formation}

In the winter of 2007, the BPXA-DOE-USGS Mount Elbert Gas Hydrate Stratigraphic Test Well on the North Slope of Alaska was drilled. This test well was a joint effort among the US Department of Energy, BP Exploration, and the US Geological Survey. ${ }^{1}$ Several hundred core samples and a variety of well-log information were collected during the expedition. A portion of the sediment samples collected was transferred to the NETL site in Morgantown, WV. Tests performed on these samples include grain size analysis and X-Ray diffraction (XRD) for mineralogy.

A summary of the data is presented in graphical form in a Lithostratigraphic Montage ${ }^{2}$. A portion of the montage is shown in Figure 1. The areas circled identify the location of methane hydrate formations. The montage also contains data on visual description, grain size, and well-bore measurements. 


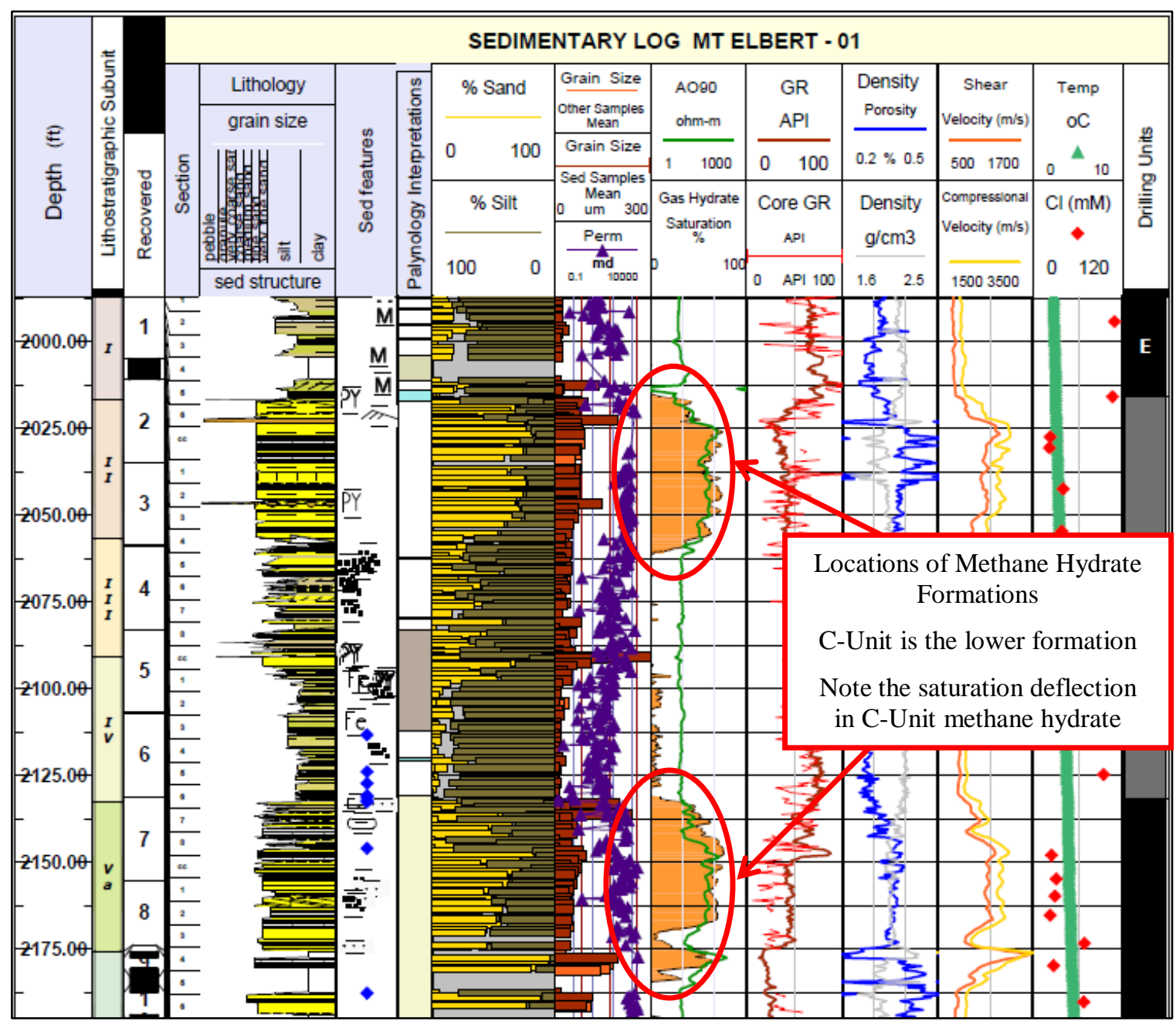

Figure 1: Lithostratigraphic Montage of Mount Elbert, AK Reservoir

Two significant intervals of methane hydrate-bearing sand-rich strata were identified within this formation. The upper interval (known as the D-Unit) begins at a depth of approximately $614 \mathrm{~m}$ below the surface and continues to a depth of approximately $628 \mathrm{~m}$ below the surface. The lower interval (known as the C-Unit) begins at a depth of approximately $649 \mathrm{~m}$ and continues to a depth of approximately $667 \mathrm{~m}$.

In the C-Unit methane hydrate interval in the Mount Elbert Reservoir, the methane hydrate saturation suddenly goes to zero (this will henceforth be referred to as a 
"saturation deflection") over an interval from $661 \mathrm{~m}$ to $663 \mathrm{~m}$ below the surface. From Rose, et al, $2011^{2}$, there is no obvious change in lithology or other apparent reason for the methane hydrate saturation deflection. Below the saturation deflection, another section of methane hydrate is found from a depth of $663 \mathrm{~m}$ below the surface to a depth of $665 \mathrm{~m}$. This unusual relationship began to draw attention from within the methane hydrate research community.

The saturation deflection is unusual because the requirements for forming methane hydrate exist in the area, yet none is present. The area has sufficient gas saturation, the sediment is of appropriate porosity, the area is within the methane hydrate stability zone, and water is present. The saturation deflection is sandwiched between layers of hydrate above and below it. A more detailed look at the sediment samples is expected to show some possible evidence for its existence. 


\section{$3 \quad$ Analysis of Mount Elbert Sediments}

As part of the Lithostratigraphic analysis of subsamples from the Mount Elbert cores, three types of analyses were conducted. These included grain size, composition by XRD, and optical microscopy. ${ }^{2}$

\subsection{Grain Size Analyses}

The first test to be completed on the methane hydrate-bearing sediments was grain size analysis. The analytical instrument used was a Malvern Mastersizer 2000. This instrument uses laser diffraction and self-contained software to complete three measurements of particle size distribution and provide a summarized average of grain $\operatorname{size}^{3}$. A summary of the grain size data from the C-Unit formation is listed in Table 1 (from Rose, et al, $2011^{2}$ ). The sample analysis procedure used at NETL for grain size is included as Appendix 1. 
Table 1: Grain Size via Laser Diffraction (from Rose, et al, 2011²)

\begin{tabular}{|c|c|}
\hline Depth, meters & Average Grain Size, microns \\
\hline 656.0 & 88.4 \\
\hline 656.4 & 56.5 \\
\hline 656.8 & 97.9 \\
\hline 657.0 & 89.1 \\
\hline 657.3 & 76.6 \\
\hline 658.1 & 78.8 \\
\hline 658.3 & 72.3 \\
\hline 658.7 & 70.9 \\
\hline 658.9 & 55.3 \\
\hline 659.3 & 62.1 \\
\hline 659.7 & 58.6 \\
\hline 659.9 & 70.6 \\
\hline 660.3 & 72.2 \\
\hline $\mathbf{6 6 1 . 3}$ & $\mathbf{6 7 . 0}$ \\
\hline $\mathbf{6 6 2 . 2}$ & $\mathbf{5 8 . 1}$ \\
\hline $\mathbf{6 6 2 . 5}$ & $\mathbf{5 6 . 9}$ \\
\hline 663.6 & 57.8 \\
\hline 664.7 & 228.4 \\
\hline 667.7 & 21.4 \\
\hline
\end{tabular}

Samples from within the methane hydrate saturation deflection are highlighted in the table. The grain size data shows no particular difference between hydrate bearing and non-hydrate bearing sediments.

\subsection{X-Ray Diffraction Analyses}

Semi-quantitative compositional analysis was completed via XRD. The instrument used to complete the analysis was a Terra InXitu portable $\mathrm{XRD}^{4}$. The XRD patterns were interpreted using MDI Jade Version 9 software $^{5}$. The results of the analyses for the CUnit hydrate formation are listed in Table 2 (from Rose, et al, 2011 ${ }^{2}$ ). The sample analysis procedure used at NETL for XRD is included as Appendix 2. 
Table 2: Composition via X-Ray Diffraction (from Rose, et al, 2011²)

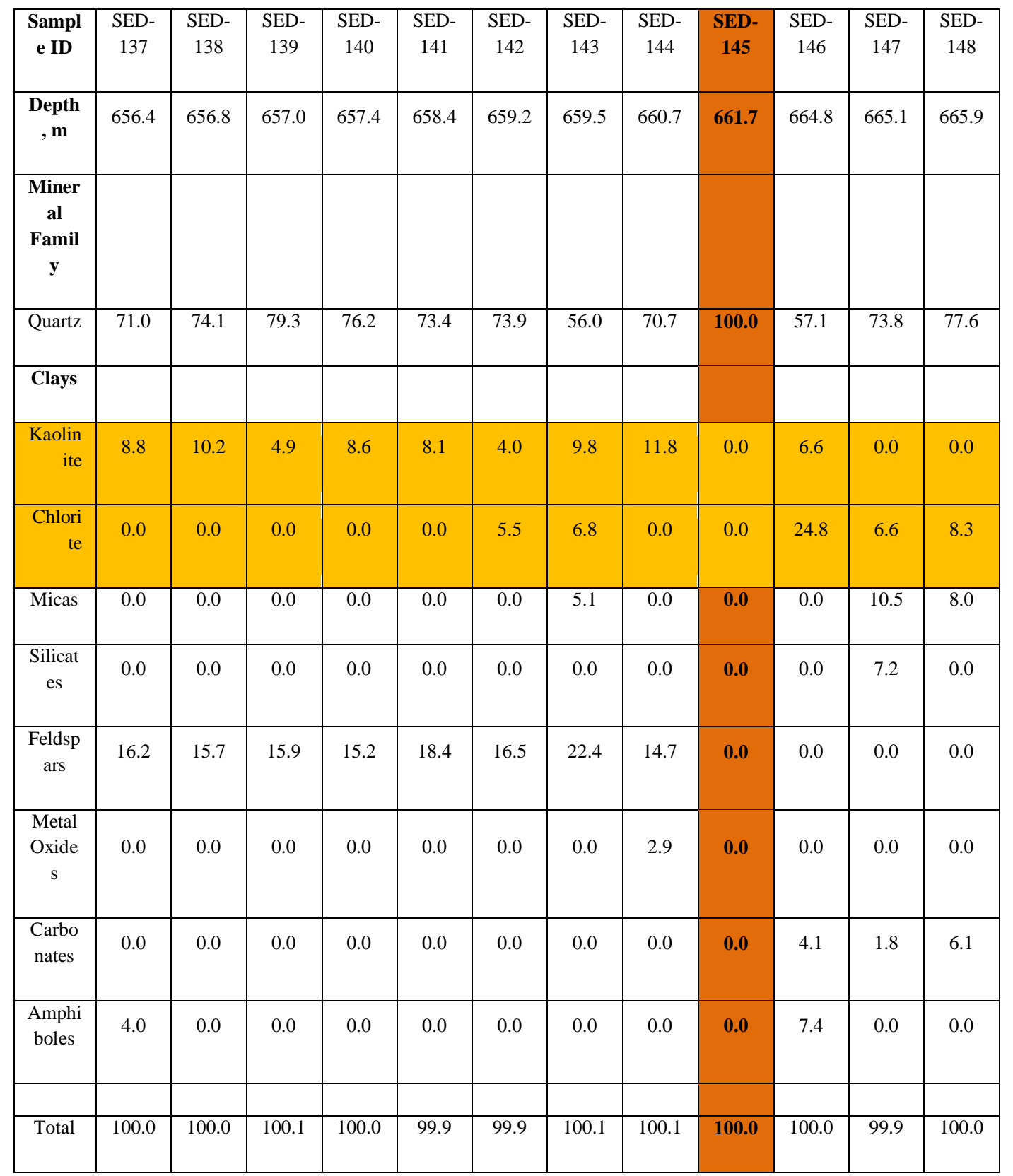

Note: The total of the individual mineral compositions for a particular sample may be $\pm 0.1 \%$ of $100 \%$. This is due to rounding error on the compositional data analysis tool - MDI Jade software.

This data in Tables 1 and 2 reveal a potential correlation between composition and hydrate saturation, but not grain size. The sediment sample located at a depth of $661.74 \mathrm{~m}$ below surface has a composition of $100 \%$ quartz. The samples in the surrounding 
locations all contain some form of clay. The minerals highlighted in the table - chlorite and kaolinite - are two varieties of clay. 


\subsection{Scanning Electron Microscopy Analyses}

In addition to petrographic microscopy, higher resolution imagery of select samples by Scanning Electron Microscope (SEM) was performed in support of this study. Within the Department of Chemical Engineering at West Virginia University resides a Hitachi S4700 SEM capable 30X - 500,000X magnification. Samples imaged in this study were analyzed at a voltage of 5 kilovolts $(\mathrm{kV})$ under high vacuum $\left(10^{-8}\right.$ torr). A standard distance of $12 \mathrm{~mm}$ from the tip of the electron emitter to the sample was used. Samples were thoroughly dried in an oven at $70^{\circ} \mathrm{C}$ for a minimum of 48 hours to remove moisture. The samples were sputter-coated with 4-6 nanometers of gold/palladium prior to imaging to ensure a conductive surface.

The procedure used for preparing and imaging samples in the SEM is documented in Appendix 3.

Select samples from the Mount Elbert, AK Stratigraphic Test Well were imaged with this device. The samples chosen for SEM imaging were identified by their Mount Elbert expedition identification numbers; SED-141 (660.35 m below surface) and SED-145 (663.74 $\mathrm{m}$ below surface). SED-141 was chosen because it was located within the C-unit hydrate formation. SED-145 was chosen because it was located within the saturation deflection.

\subsubsection{SED-141 Images}

The SEM images in Figures 2 and 3 for SED-141 location (approximately $658.35 \mathrm{~m}$ below the surface) show quartz particles, greater than $100 \mu \mathrm{m}$, coated with clay. The clay 
adheres to the surface of the quartz particle, even when thoroughly dried and imaged under high vacuum.

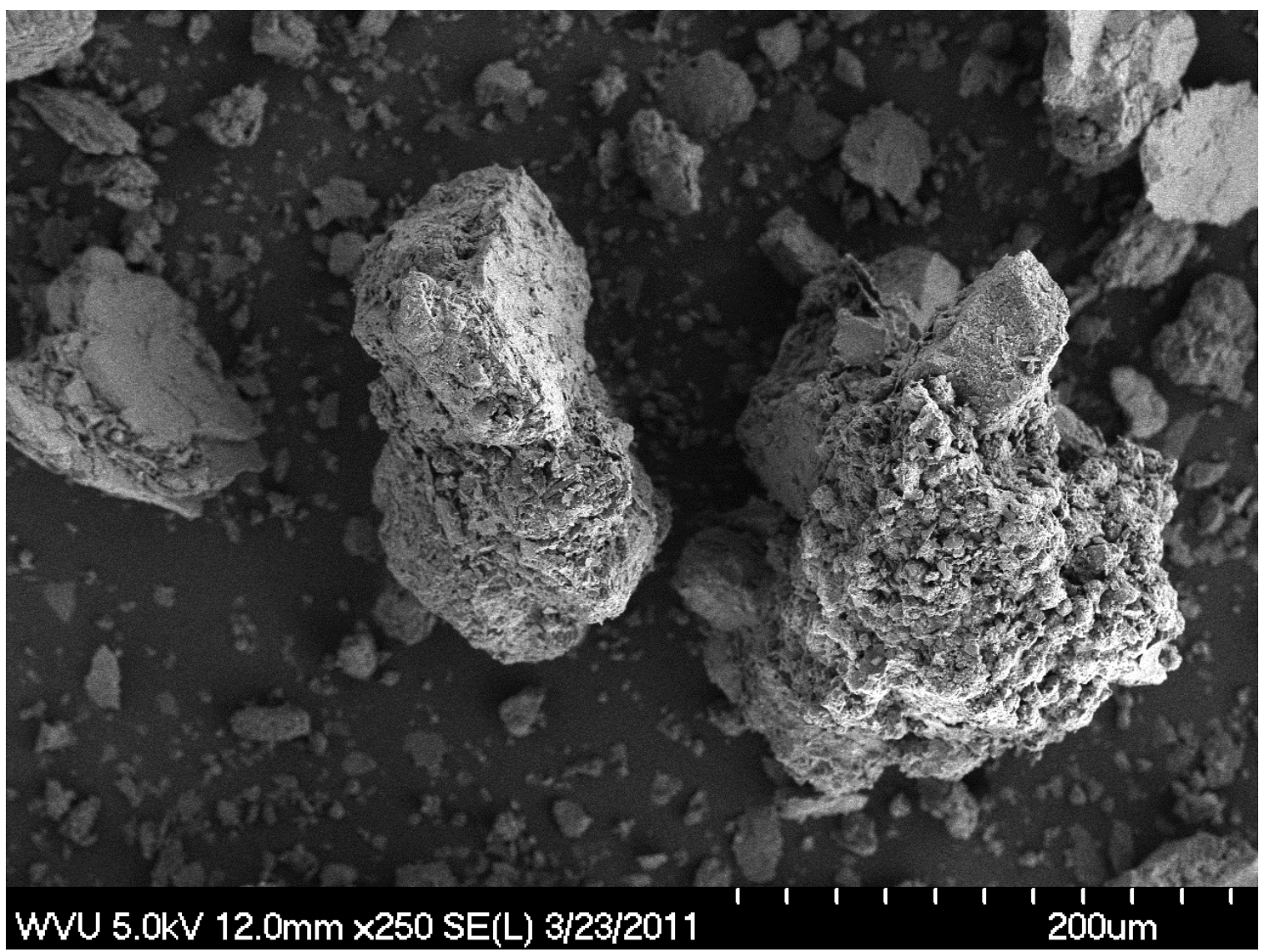

Figure 2: SED-141 Image 1 - Sample at 250X magnification. Note the clay coating, which gives the coated quartz grains a fuzzy appearance. Particles of clay and small quartz grains can be seen in the background on the mounting tape. 


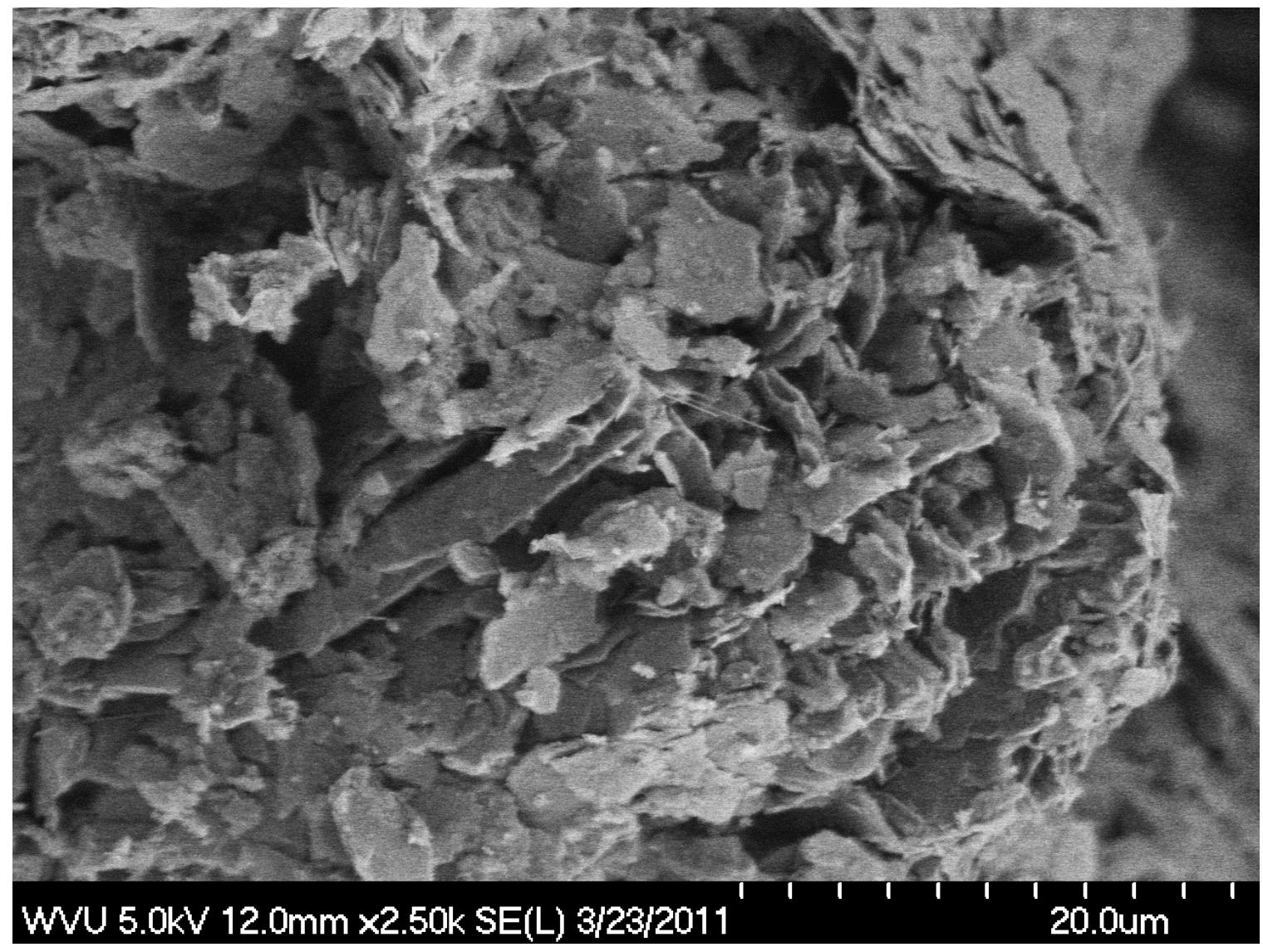

Figure 3: SED-141 Image 2 - Sample at 25,000X magnification. Individual grains of clay can be seen adhering to the quartz grain and to other clay particles.

\subsubsection{SED-145 Images}

The SEM images in Figures 4 and 5 for SED-145 location (approximately $661.74 \mathrm{~m}$

below the surface) show greater than $100 \mu \mathrm{m}$ quartz particles with limited or no coating.

Smaller quartz fines are present in the sediment, but do not adhere uniformly to the larger particles. 


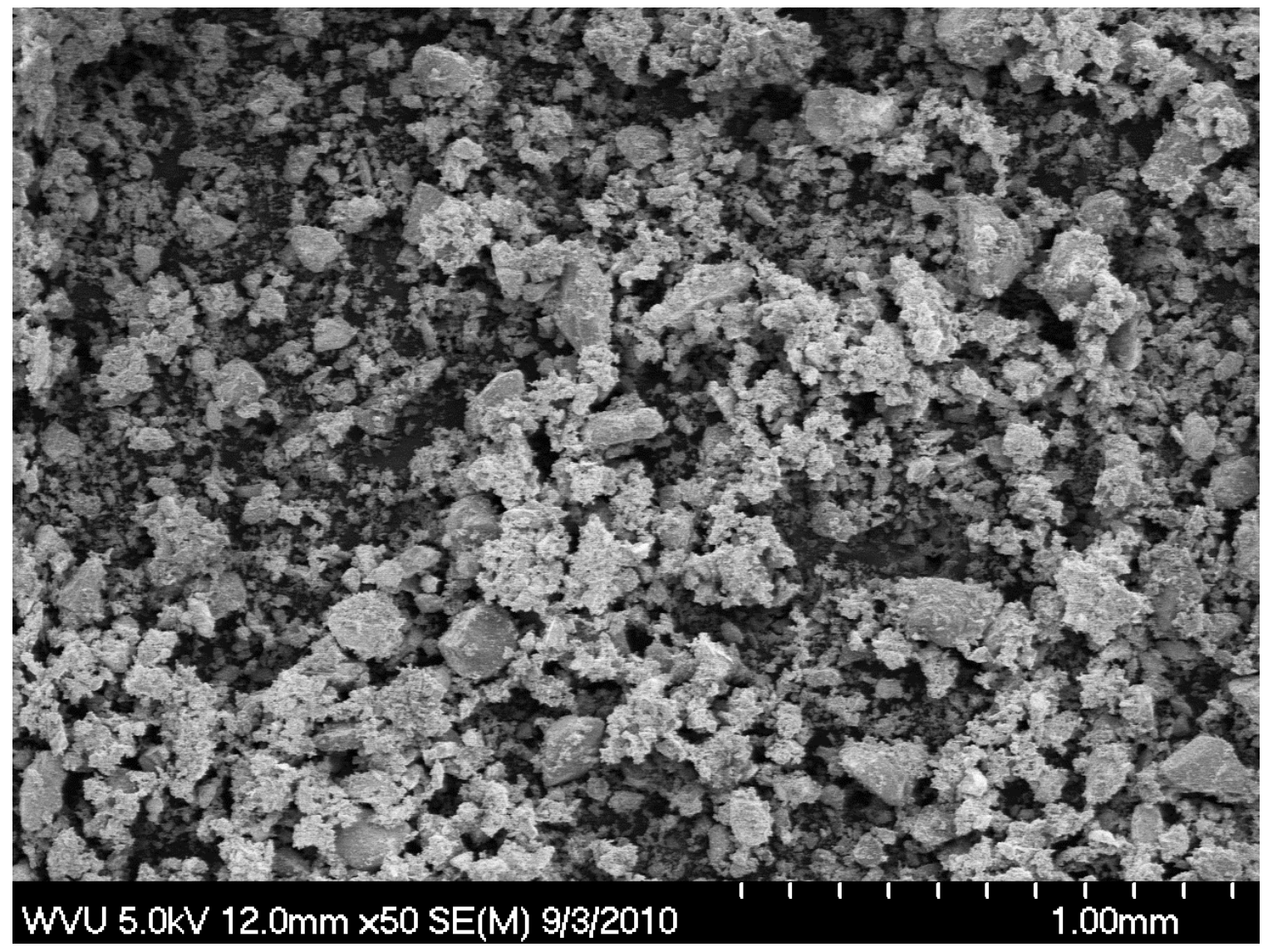

Figure 4: SED-145 Image 1 - Sample at 50X magnification. On several of the larger grains, flat surfaces can be seen. Random agglomeration of smaller fines can be seen throughout the image. 


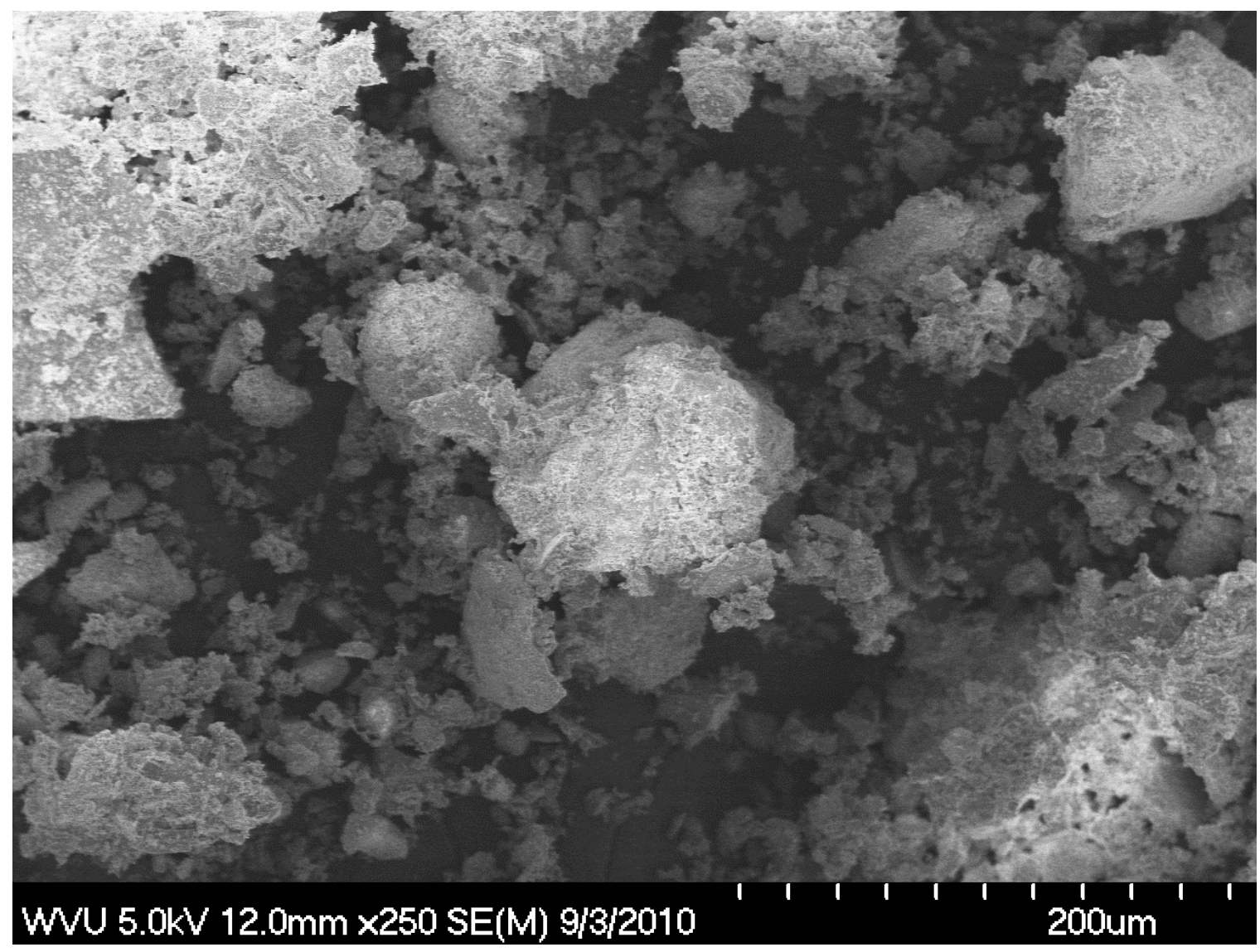

Figure 5: SED-145 Image 2 - Sample at 250X magnification. The fine grains do not adhere uniformly to the larger quartz grains.

Imaging of the SED-145 sample at higher magnifications was not possible, as the surface would "glow" in the SEM. Glowing is an indication of a non-conductive material, even though the sample was coated with the gold/palladium. Glowing was not an issue in the SED-141 sample, which was prepared identically to SED-145. 


\section{Development of Hypothesis Concerning Sediment Composition}

The hypothesis that sediment composition played a key role in the experimental formation of methane hydrate was developed through a review of Mount Elbert reservoir data.

\subsection{Review of Observations concerning Mount Elbert Reservoir}

The sediment samples analyzed from the Mount Elbert methane hydrate formation show a particle size that is non-uniform. The average particle size in the hydrate formation ranges from $56 \mu \mathrm{m}$ to $228 \mu \mathrm{m}$. This average particle size represents a weighted average of all grain sizes from within a sediment sample. Each sample will contain a fraction of material in the three size ranges of the Wentworth grade scale - clay (less than 3.9 micron), silt (3.9 - 62.5 micron), and sand (greater than 62.5 micron - 2000 micron) ${ }^{6}$. A variance in average particle size does not seem to explain the saturation deflection.

On the other hand, the compositional data show a different story. In the areas where hydrate has formed, approximately $10 \%$ of the sediment is comprised of clay. The typical clay found in the Mount Elbert $\mathrm{C}$ and $\mathrm{D}$ Unit sediments is kaolinite $\left(\mathrm{Al}_{2} \mathrm{Si}_{2} \mathrm{O}_{5}(\mathrm{OH})_{4}\right)$. The remainder of the sediment is quartz $\left(\mathrm{SiO}_{2}\right)$, with other silicate minerals. For the lone sample in the saturation deflection, the compositional analysis shows only quartz.

\subsection{Discussion of How Observations Lead to Hypothesis}

The data begs the question: Whether the lack of clay in the saturation deflection sediment indicates something that might be generalizable to other hydrate deposits? Clay, as a 
water-absorbing mineral coats the surface of quartz and other minerals (even when wet) in the Mount Elbert sediment. This observation results in the hypothesis: Clay may act as a distribution material for water and/or methane during the formation of methane hydrate in sediment. If the clay helps distribute and maintain concentrations of water and/or methane, then the resultant methane hydrate would be more uniform than without it. The difficult task of bringing two dissimilar forms of matter (liquid water and gaseous methane) together to form a third state of matter (solid hydrate) may be aided by the presence of clay.

\subsection{Sediment Composition Plays an Important Role in Hydrate Formation}

The previous data review and analysis lead to the following hypothesis:

For the purposes of experimental methane hydrate formation in packed sediment, it is hypothesized that the composition of the sediment plays a vital role in the uniformity of the methane hydrate formed within the sediment. The wetted clay may stabilize the water and/or methane concentration throughout the sediment, thus creating an environment by which a uniform methane hydrate formation may result. Grain size (within the limits of the Mount Elbert sediments) is not expected to influence how uniform the methane hydrate is.

\section{$5 \quad$ Experimental References and Current Laboratory Methodology}

Many researchers have conducted laboratory experiments on methane hydrate formation. Many studies have focused on the formation of pure methane hydrate, such as the United

States Geological Survey (USGS) ${ }^{7}$. As the work in this thesis proposal is intended to 
determine the controlling variables in a natural system, we will mostly discuss experiments that form methane hydrate in porous media.

Each laboratory focuses on a particular aspect of methane hydrate formation. However, almost universally, the various laboratories use a pure substance as the sediment base.

Work done by the Sloan research group in the Center for Hydrate Research at the Colorado School of Mines ${ }^{8}$ on methane hydrate phase equilibria was conducted in a core made from Adriatic sandstone (nearly pure quartz). Their goal was to measure phase equilibrium shifts with respect to pore size. The authors observed a shift in observed data from predicted values. The reason for the shift was unclear, but they postulated that sediment type may be a contributor.

Another experiment conducted by $\mathrm{Lu}$, et $\mathrm{al}^{9}$ focused on the effect of particle size on the saturation of methane hydrate. Their experimental work was conducted using silica sand with a purity of $>99 \%$ and various particle sizes. The researchers note that natural sediments contain complex constituents, but do not pursue what role these other constituents may play.

The formation of artificial methane hydrate for the purposes of storage and transport has interesting industrial potential. Ganji, Manteghian, and Mofrad ${ }^{10}$ conducted experiments on the use of surfactants in accelerating the formation of methane hydrate. This work was not conducted in sediment. However, this work is noteworthy because they pursue the effect that something other than methane and water in a generic base may impact how methane hydrate forms. While their work did show that the surfactants improve the rate 
at which the methane hydrate formed, the surfactants also accelerated the decomposition rate.

Prior studies have not totally ignored the effect that clay may play in the formation of methane hydrate. Reistenberg, et al $^{11}$ conducted a study using bentonite (a swelling clay of impure composition) and silica suspensions. Their work showed that the presence of bentonite reduces the overpressure needed to form methane hydrate in a colloidal suspension. However, their work was not done in packed sediment. If bentonite were to be used in packed sediment, the swelling experienced upon hydration would more than likely block water and gas flow through the sediment.

Zeolite is an alumino-silicate mineral that has some properties similar to clay. Zang, Du, Liang, Fan, and Tang ${ }^{12}$ conducted experiments using A-type zeolite as a base for methane hydrate formation. The difference between zeolite and clay lies in the mineral structure. Clays are plate-like structures where water is absorbed into the mineral matrix ${ }^{13}$. A-type zeolite has a porous but rigid structure, where water distributes itself over the surface of the mineral matrix ${ }^{14}$. The A-type zeolite provided for steady formation of methane hydrate in their experiments, which was attributed to the porous surface of the zeolite particles.

An experiment which involved sediment similar to the proposed work was conducted by Zhang, et $\mathrm{al}^{15}$. The experiments used loess, which is a sedimentary deposit of silt-sized grains, loosely consolidated by calcium carbonate. ${ }^{16}$ Loess typically contains $5-10 \%$ of clay particles, depending on its source. Loess does not have a defined composition. Zhang and co-workers placed unpacked loess in the base of a pressure vessel with an 
amount of water and then maintained a constant pressure of methane. This allowed methane hydrate to form in the loose sediment. They then calculated the conversion of the methane and water to methane hydrate. The experiments resulted in calculated formation rate data for methane hydrate formation in loess.

Seol and Kneafsey ${ }^{17}$ used a sediment core packed with various sizes of silica sand to form methane hydrate. During the formation of methane hydrate, water was imbibed more readily into the fine grained section of the sediment core. This may be partially explained by surface tension effects in the sediment. The finer grain sections will have a greater surface area, and thus attract more water via surface tension. The methane hydrate formed in the experiments was imaged with a CT scanner. The methane hydrate formations were found to be highly irregular.

From these reported experiments, it can be seen researchers have a desire to reproduce what is known to occur in nature. However, in order to simplify the experiment and reduce risk to expensive equipment, simple sediments have been chosen as a base for the formation of methane hydrate. The literature hints at the potential that clay may act as a catalyzing factor in hydrate formation, but to date the issue has not been addressed.

This study presents a method for preparing artificial sediments that have many of the same characteristics of natural sediments. The artificial sediments will then be used in the preparation of methane hydrate and imaged with CT scanning to verify the formation and its uniformity. If the hypothesis is correct, the very first uniform artificial methane hydrate formation may occur. 


\section{Description of Experiment to Test Hypothesis}

An experiment was designed to test the theory that sediment composition plays a role in the uniformity of distribution of methane hydrate formed in packed sediment. Novel methods for sediment preparation and compaction were developed. These methods sought to replicate the natural system as close as possible. To mimic natural sediments, the grain size and composition of core samples from the Mount Elbert, Alaska well were reviewed, and were used as a guide.

The equipment available for experimentation was located in both the National Energy Technology Center (NETL) in Morgantown, WV and at West Virginia University (WVU) in Morgantown, WV. Sample preparation and packing occurred at WVU. Methane hydrate formation experiments occurred at NETL.

\subsection{Preparation of Artificial Sediment Samples}

The equipment required for sediment preparation was typical laboratory equipment beakers, stirring rods, a gram scale, and an oven.

Artificial sediment was prepared by mixing fine particles (approximately 1 micron kaolinite or ground quartz) with larger quartz granules (approximately 130 micron) in water, and then drying the mixture. This procedure allowed the fine particles to distribute throughout the larger quartz grains.

The large grain quartz used in these experiments was F110 sand - manufactured by US Silica and provided by NETL. The average grain size was $130 \mu \mathrm{m}$. The manufacturer's 
analysis and XRD performed at NETL showed the material to be virtually $100 \%$ quartz.

The kaolinite used was another product of US Silica. XRD analysis at NETL showed the material to be nearly pure kaolinite. The average grain size of the kaolinite was $1 \mu \mathrm{m}$.

The fine grained quartz was obtained from US Silica and XRD analysis at NETL showed the material to be nearly pure quartz. The average grain size of the ground quartz was 1 $\mu \mathrm{m}$.

\subsubsection{Preparation of Large Grain Quartz/Kaolinite Artificial Sediment}

The primary artificial sediment used in experimentation was a mixture of kaolinite with quartz. The goal of the sediment preparation was to achieve an artificial that mimicked natural sediments in appearance. The tool used for visual comparison was the SEM. The following steps detail the preparation of the artificial sediment.

\subsubsection{Weighing and Mixing}

From the data presented in Table 2, the approximate average percentage of clay in the Mount Elbert, AK sediment is $10 \%$. This was established as a standard for the preparation of artificial sediments.

In a 1-liter beaker, 180-grams of F110 Sand were weighed. Added to the F110 Sand were 20-grams of kaolinite clay. The kaolinite clay had been previously analyzed by XRD and found to be nearly pure kaolinite. The 200-gram mixture was wetted with approximately 500-milliliters of deionized water (DI Water). The wetted mixture was thoroughly stirred to wet the components. 


\subsubsection{Drying}

The beaker with the wetted mixture was placed in a $70^{\circ} \mathrm{C}$ oven to dry. Every 6-12 hours, the mixture was stirred with a rod to break up clumps and surface skin. The mixture was determined to be dry by comparing the weight of the individual components to dried mixture. Within 2-3 days, the added water had evaporated.

\subsubsection{Breaking of Clusters}

Large clumps or clusters were formed during the drying of the artificial sediment. In order to break the clusters, light grinding with a mortar and pestle placed in the same oven as the sediment was done. The warm mortar and pestle was used to prevent moisture from being re-absorbed by the clay. Care was taken not to grind too vigorously, as this would affect the general particle size of the quartz grains.

\subsubsection{Second Drying}

After breaking down the clusters, the mixture was placed back in the beaker and put back in the $70^{\circ} \mathrm{C}$ oven for 2-3 days to allow any residual water to evaporate from the artificial sediment. After the second drying, the artificial sediment was ready to use.

\subsubsection{Preparation of Large Grain Quartz/Ground Quartz Artificial Sediment}

For comparison, a second artificial sediment was prepared by replacing the kaolinite with ground quartz of approximately $1 \mu \mathrm{m}$ particle size. This allowed for experimentation that detailed any effects based solely on composition, and excluded the possibility that grain size is a factor in controlling hydrate saturation and distribution. The ground quartz/large 
grain quartz mixture was prepared identically to the kaolinite/large grain quartz mixture.

\subsubsection{Visual Comparison of Kaolinite/Quartz versus Ground Quartz/Quartz Sediments}

The kaolinite/quartz sediment formed larger and harder clusters than the ground quartz/quartz sediment. The kaolinite/quartz sediment clusters showed that an affinity existed between the clay and quartz that did not exist between ground quartz and largergrain quartz particles.

\subsubsection{SEM Imaging of Prepared Sediments}

SEM images were taken of the prepared sediments to compare them to the natural samples taken from the Mount Elbert, AK well. The images showed that the sample preparation technique provided an artificial sediment which visually compared closely with the natural sediment.

Figure 6 shows F110 sand grains, for use in comparison to the mixed samples presented in later figures. Figures 7 and 8 show the kaolinite clay has thoroughly and uniformly coated the surface of the larger quartz granule. 


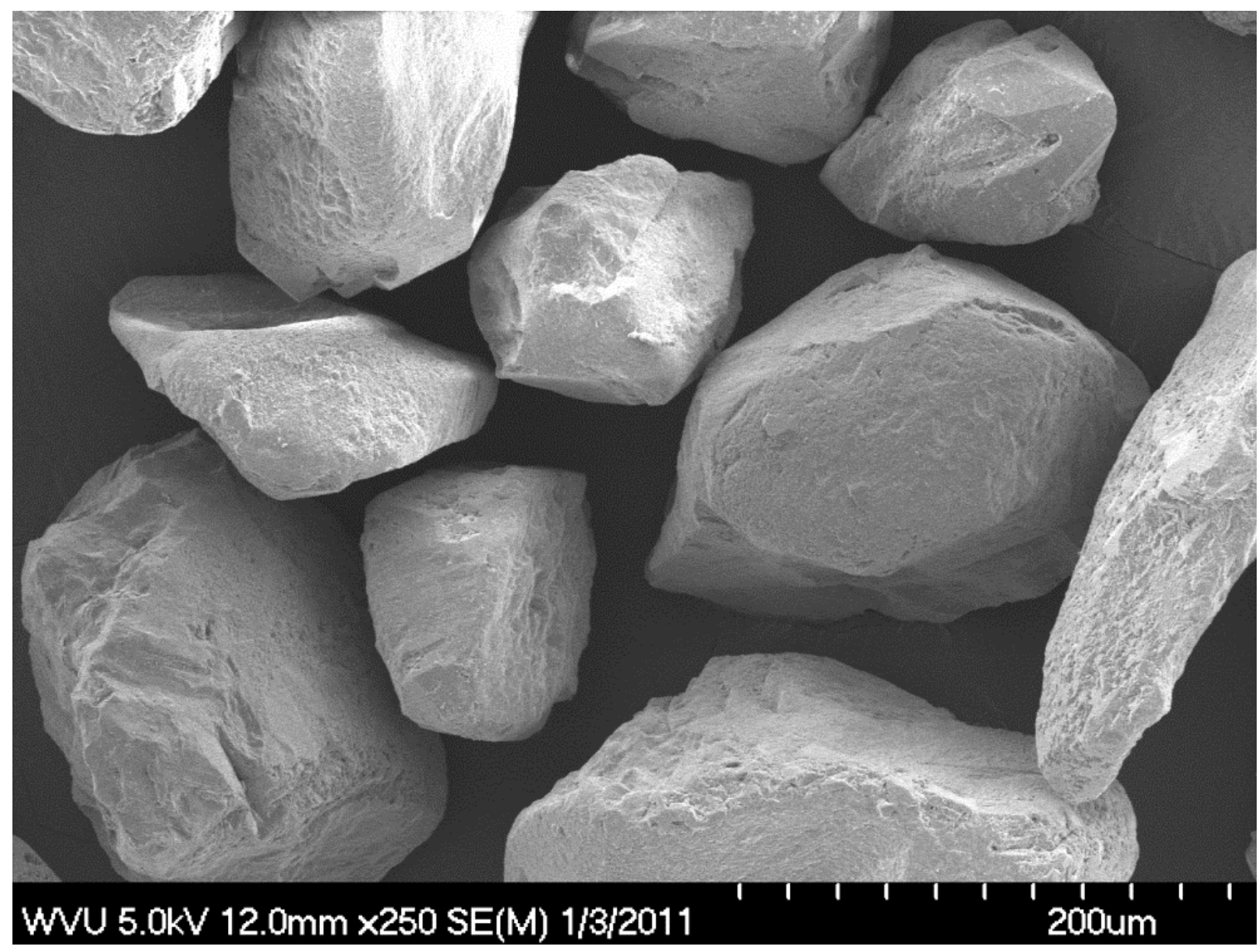

Figure 6: 250X Magnification of F110 Sand Grains as received from US Silica

The surface of the F110 sand grains in Figure 6 showed a rough texture. Any coating on the surface of these grains obscured this rough texture. 


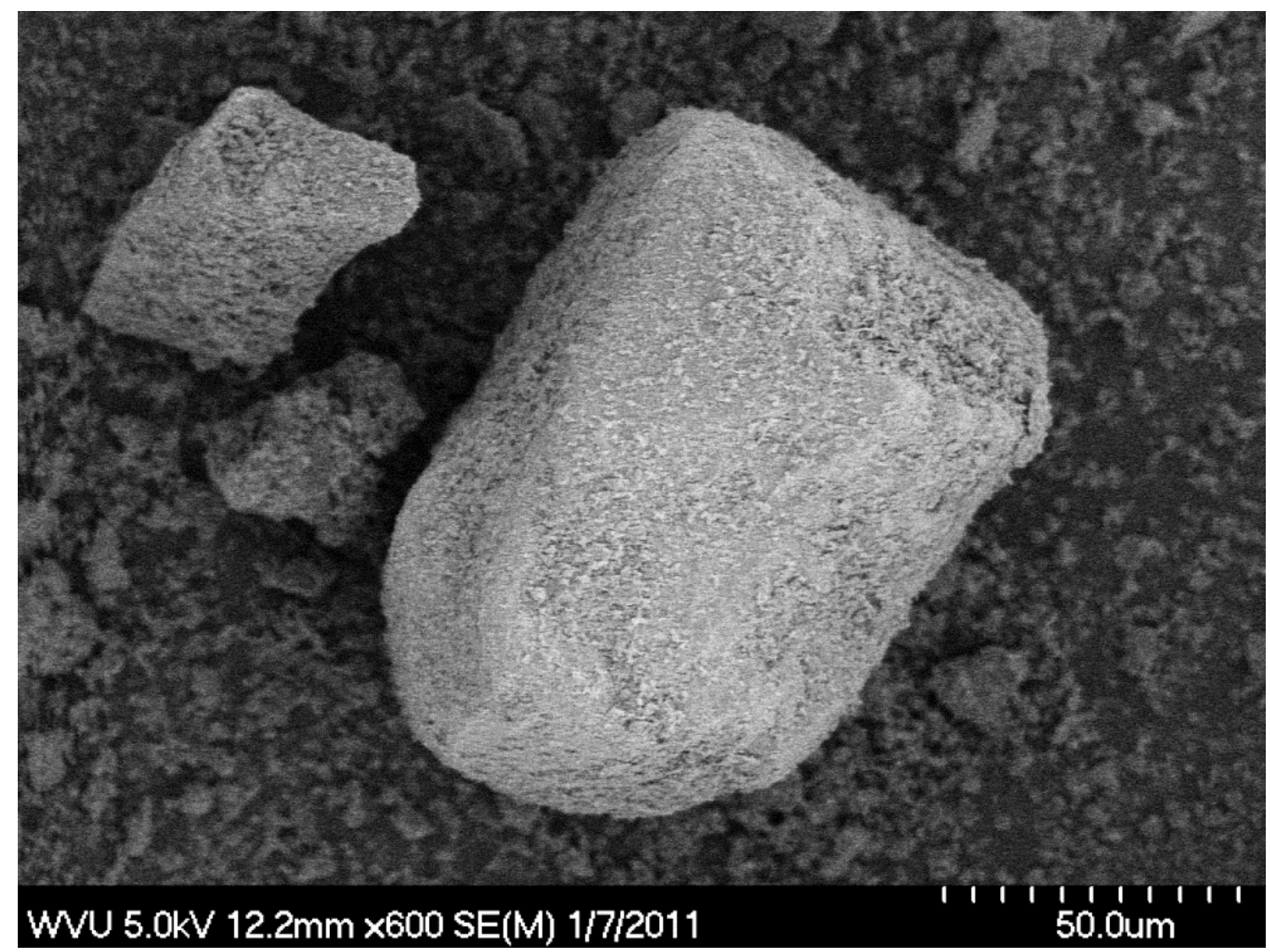

Figure 7: 600X magnification of quartz/kaolinite artificial sediment showing fuzzy texture, which is an indication of uniform coating of the large quartz grain

A fuzzy surface coating of kaolinite clay was present on the larger quartz grains in Figure

7, which made the surface appear to be smoother than it was. In comparison, the lack of coating on the quartz grains in Figure 9 made this more evident. 


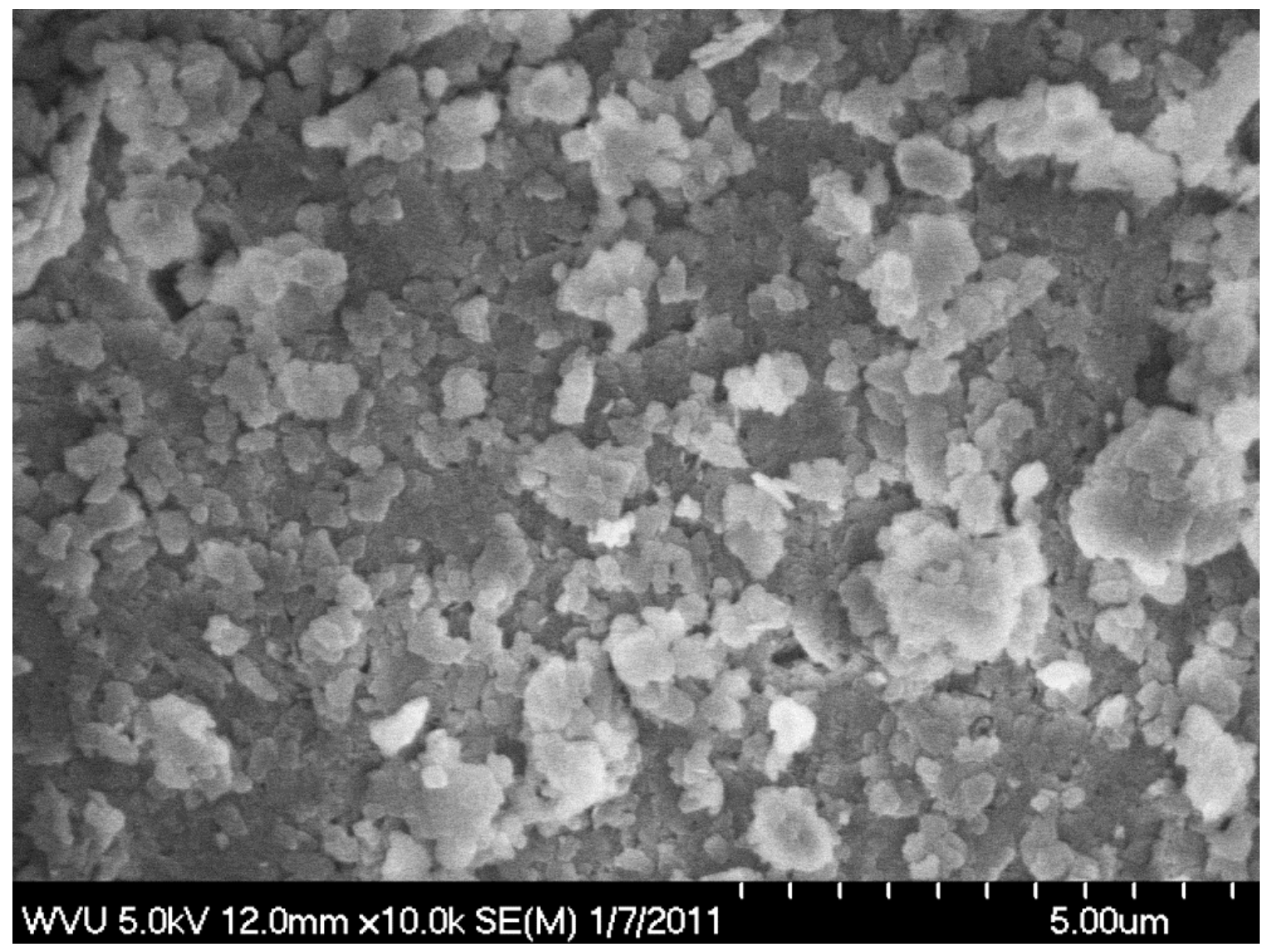

Figure 8: 10,000X magnification of kaolinite coating on the surface of the large grain quartz, showing individual grains of kaolinite clay layered on the surface

The image in Figure 8 shows individual grains of clay on the surface of the larger quartz grain.

Figures 9,10 , and 11 show that the quartz fines were distributed unevenly across the surface of the larger-grained quartz. The quartz fines had no affinity for attaching to the larger grained quartz. The quartz fines appeared to collect in ridges and valleys on the surface of the large grained quartz. 


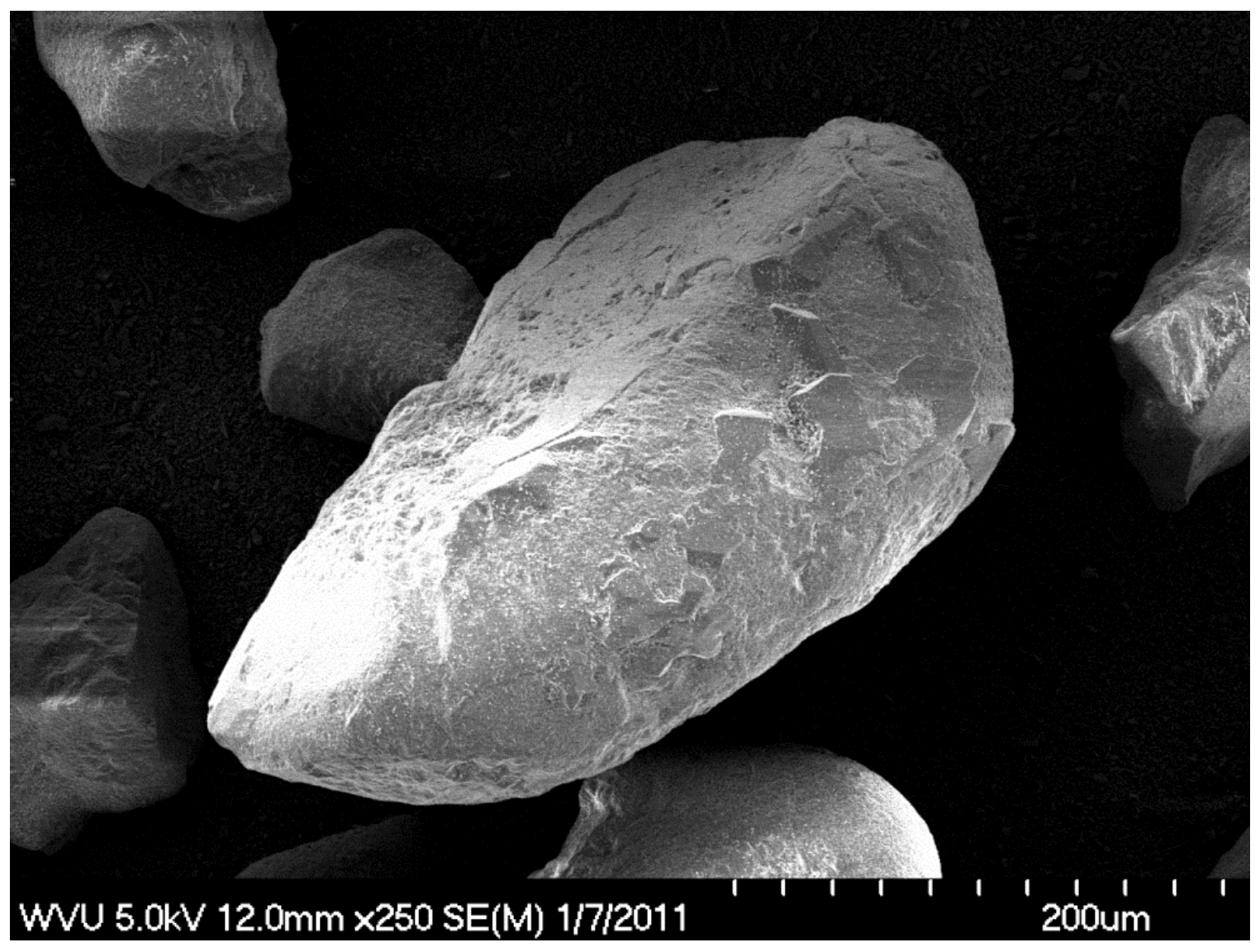

Figure 9: 250X magnification of quartz fines on large grained quartz. Note the surface detail of the grain, which indicated the lack of coating of fine grain quartz 


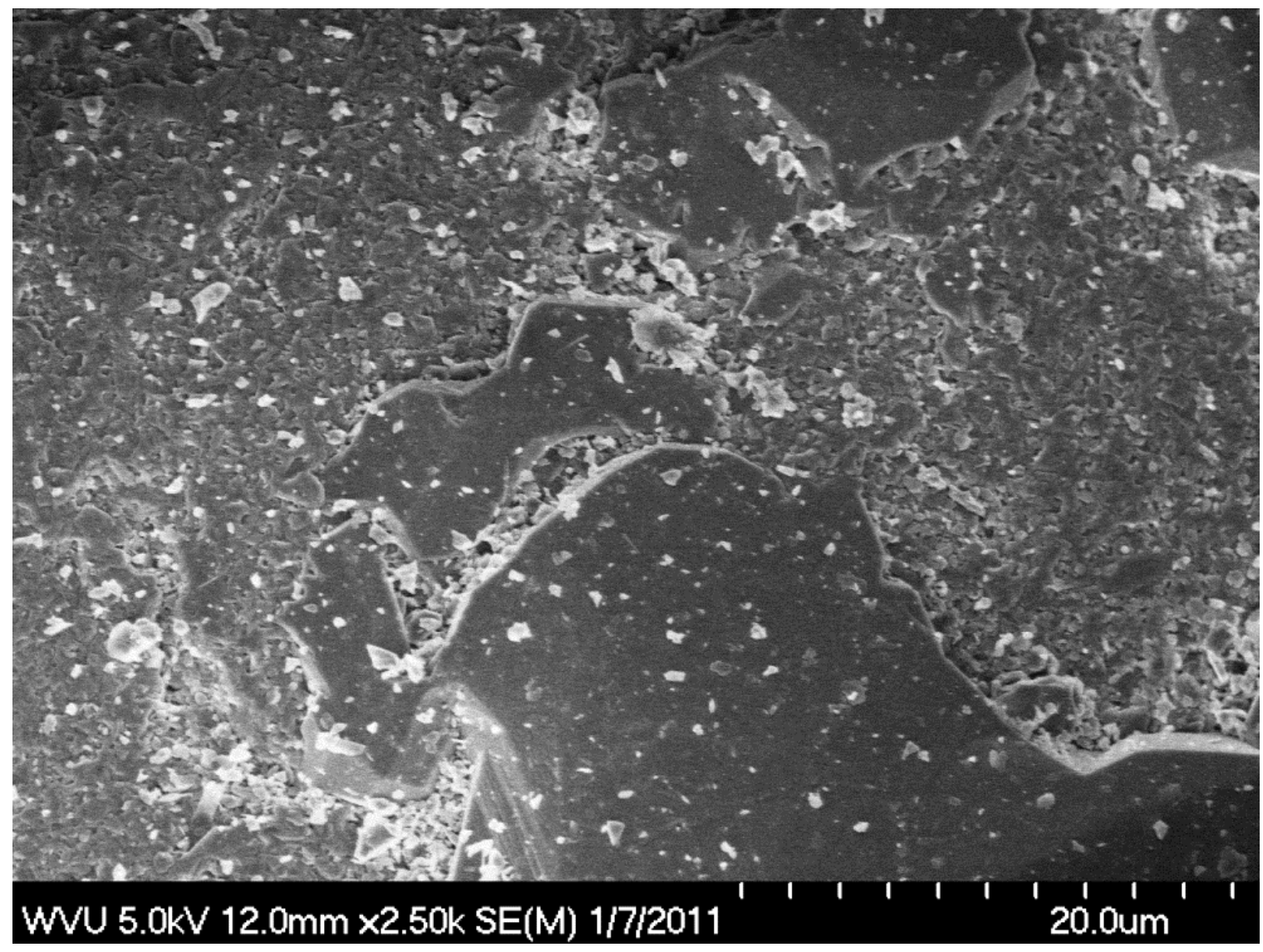

Figure 10: 2,500X magnification of quartz fines on large grain quartz. Note the sparse coating of quartz fines on the surface of the large quartz grain. The large quartz grain is virtually uncoated by the fine grain quartz. 


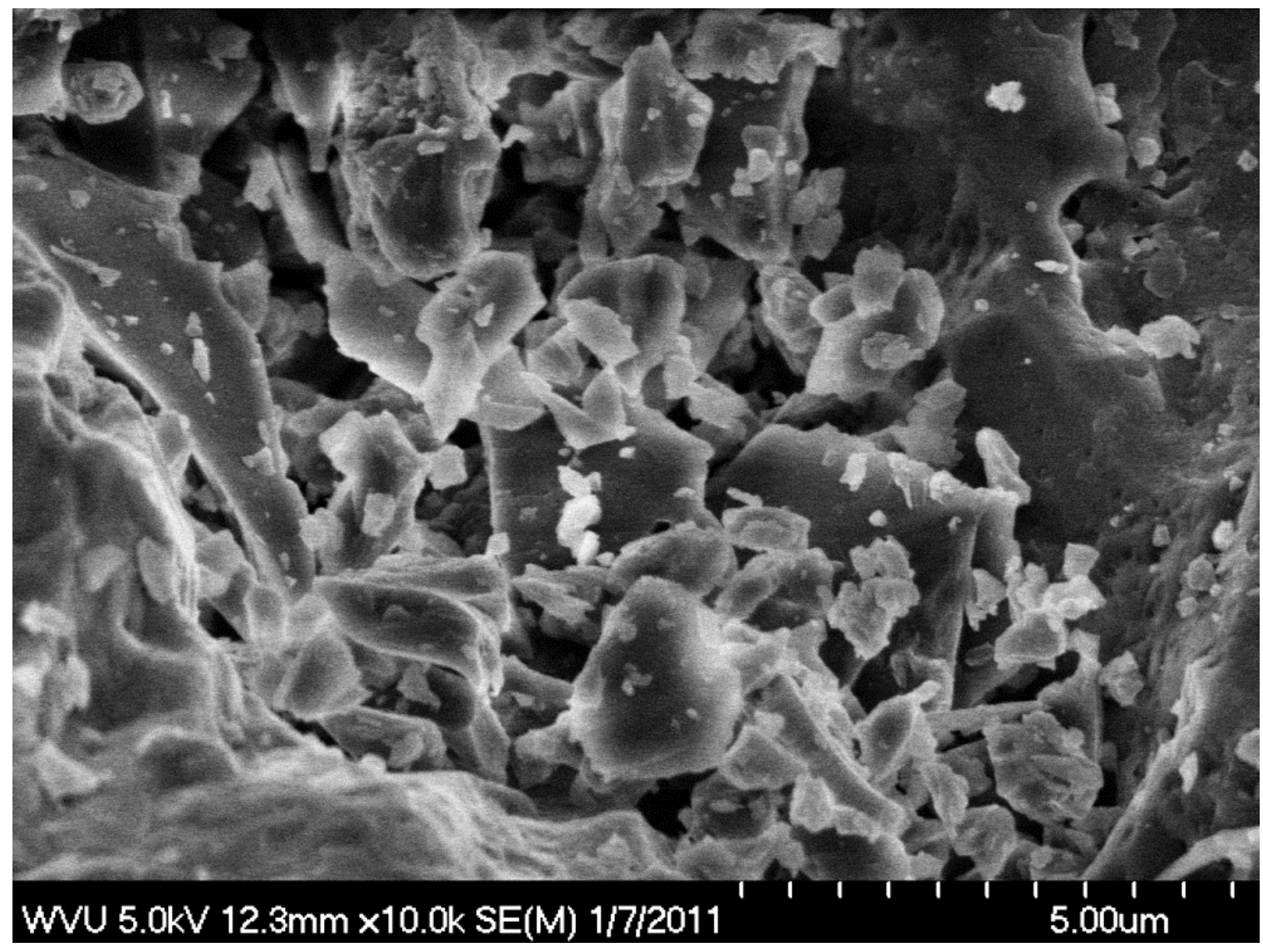

Figure 11: 10,000X magnification of quartz fines on large grain quartz surface. The $1 \mu \mathrm{m}$ fine quartz grains tended to agglomerate in irregularities on the surface of the large quartz grains. Unlike the kaolinite, the fine quartz grains did not create a uniform coating over the surface of the large quartz grain.

\subsection{Sediment Packing Procedure}

In order for the artificial sediment to be uniformly packed, a sediment packing procedure was developed. This procedure was developed using equipment at the West Virginia University Department of Chemical Engineering Carbon Products Laboratory. A Thermo Electron PR7000M floor-mounted centrifuge (shown in Figure 12) was available in the Carbon Products Laboratory at WVU. This piece of equipment is a 6-bucket centrifuge (see Figure 13 and 14) capable of programmed operation. 


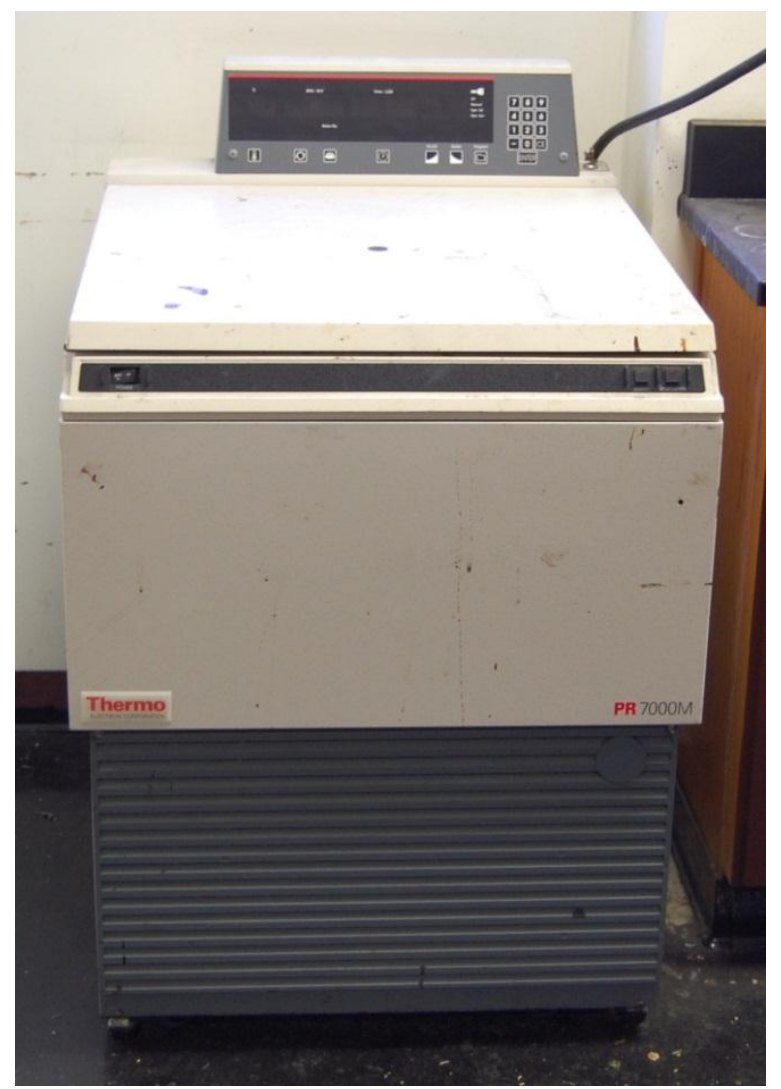

Figure 12: Thermo Electron PR 7000M Centrifuge

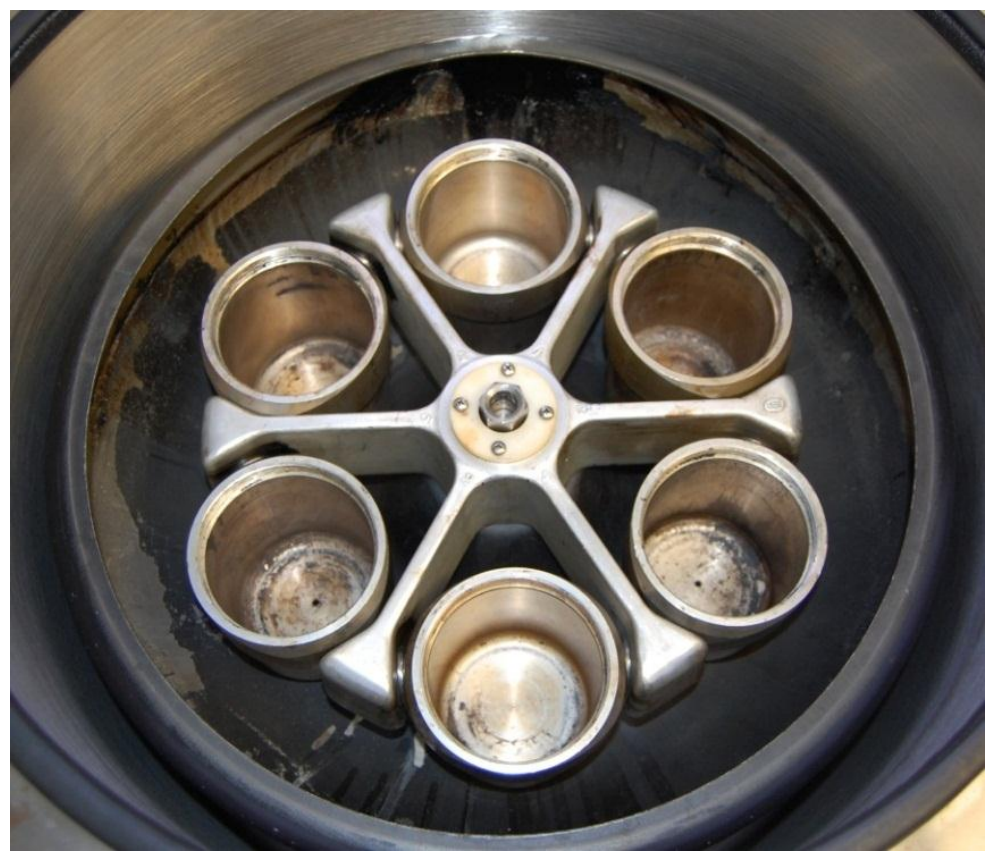

Figure 13: 6-Bucket layout of Centrifuge 


\subsubsection{Wet versus Dry Packing of Artificial Sediment}

Two methods of packing sediment were trialed - pre-wetting the sediment before placing in the Aluminum Pressure Vessel (APV) and dry packing the sediment with water addition after compaction. The most effective means for compacting the sediment without causing fines migration was dry packing.

An initial trial of wet sediment packing was centrifuged at 5,000-RPM for 60 minutes. When the wet-packed sediment was removed from the APV and visually inspected, an agglomeration of fines was observed at the bottom of the sediment core. It is also believed that the centrifugation of wet sediment caused part of the clay coating to break loose from the surface of the large quartz grains and migrate to the bottom of the APV.

More success was experienced with dry packing. When the dry-centrifuged sediment was removed from the APV and visually inspected, very little fines agglomeration was detected at the bottom. Additional time was needed to achieve the same amount of compaction. Each sample was centrifuged at 5,000 RPM for 3 cycles of 99 minutes. Dry centrifugation was chosen as the best method for packing sediment.

\subsubsection{Manufacture of Sediment Packing Equipment}

In order to properly pack the sediment, several accessories were manufactured in the West Virginia University Department of Chemical Engineering Shop. The devices manufactured were: 
6.2.1.2.1 APV Holder (shown in Figure 14) - this allowed the 1.25" outside diameter APV to be held firmly in place in the approximately 4" inside diameter centrifuge cup.

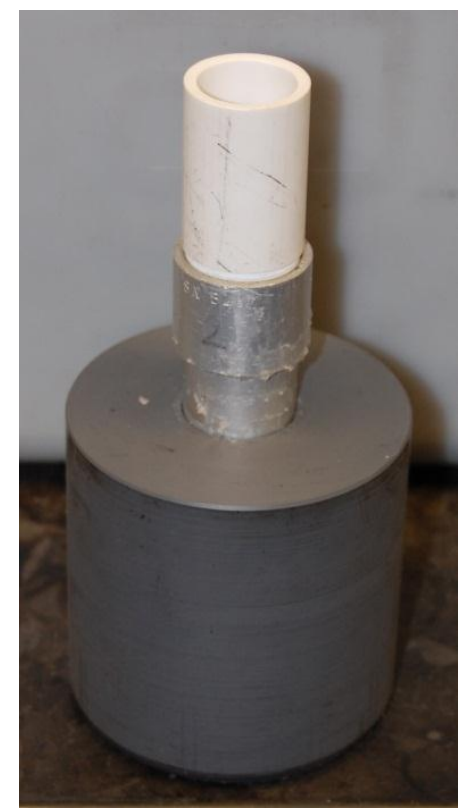

Figure 14: APV Holder with APV and Packing Guide

6.2.1.2.2 Packing Weight \& Guides (shown in Figure 15) - In order to compact the sediment in the APV with a force equivalent to the depth of the Mount Elbert saturation deflection, calculations were conducted in accordance with Newton's Second Law ${ }^{18}$. These calculations may be found in Appendix 3. The weight calculated was 161 grams.

Centrifuging with a weight of 161-grams at 5,200-RPM was determined to provide the equivalent packing force of 2,200-feet of stationary sediment with a density of 2 grams/cubic centimeter ${ }^{2}$. This was the depth of the Mount Elbert, AK methane hydrate saturation deflection. Stainless Steel rods were 
machined to slightly less than 1" outside diameter (to fit into the APV's) and a length of approximately 2 " to provide the exact weight. PVC pipe was cut to a length of 2" and threaded at one end. This was screwed into the open end of the APV to act as a guide for the packing weight.

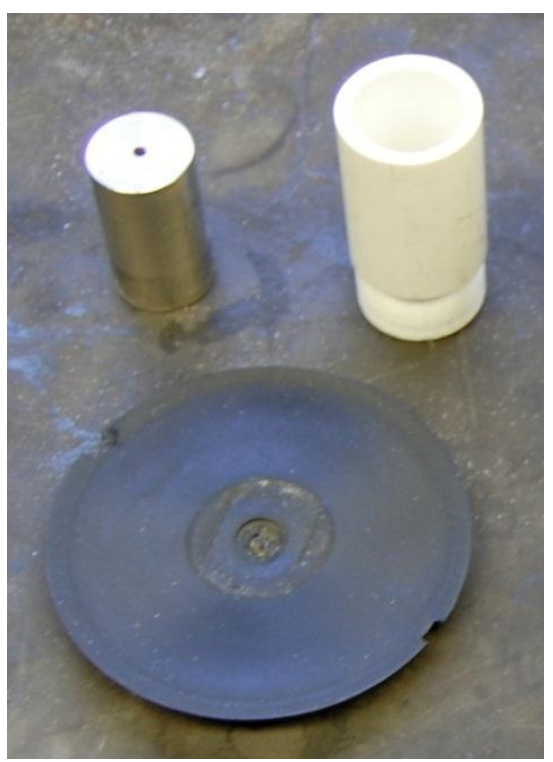

Figure 15: Packing Weight \& Guide with Rubber Base Support

\subsubsection{Final Sediment Compaction Procedure}

The final sediment compaction procedure is as follows:

1. Screw flush plug into the open base of the APV to prevent sediment from exiting APV

2. Place PVC base and sintered stainless steel plate on the bottom of the APV to support the sediment column

3. Pour 60-grams of dry artificial sediment into the APV using a funnel

4. Place sintered stainless steel plate on top of loose sediment column 
5. Screw Compacting Weight Guide into the threaded end of the APV and place Packing Weight on top of the sediment.

6. Place APV into APV Holder

7. Place APV Holder with APV into floor-mounted centrifuge.

8. Ensure a counterbalance is placed in the opposing bucket in the centrifuge. This is typically another APV with prepared sediment.

9. Close centrifuge and power on

10. Set Centrifuge parameters: Speed - 5,000 RPM, Ramp - 1 (slowest setting), Brake -5 (medium setting), Temperature $-21^{\circ} \mathrm{C}$ (ambient), Time -99 minutes (maximum allowable time). Start Centrifuge program.

11. Upon completion of Centrifuge program of 99 minutes, repeat twice, for a total of 297 minutes of centrifugation.

12. Upon removing APV from Centrifuge, remove the packing guide and weight, then screw the APV lid onto the APV to hold sediment in place.

13. Dry sediment is now ready for use.

14. If sediment is to be wetted before use, the APV can be placed on a scale and DI water added via a dropper to the desired weight.

\subsection{Visual Sediment Packing Experiments}

Polycarbonate tubes (shown in Figure 16) of equivalent diameter and length to the APV were constructed to visually observe what happens to sediment when it is compacted and hydrated. The polycarbonate tubes provided valuable insight into the hydration of dry 
artificial sediment.

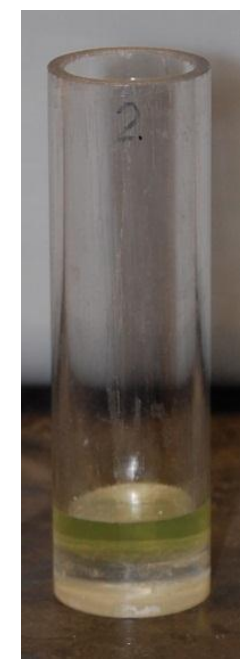

Figure 16: Empty polycarbonate tube with epoxy resin seal at the base

The polycarbonate tubes were loaded with 60 grams of dry sediment. Sediments in the polycarbonate tubes could be centrifuged. However, due to the low strength of the polycarbonate/glue construction, a maximum of 1,000-RPM could be used before physical damage occurred to the bottom seal. The dry sediment was centrifuged under a 161 gram weight at 1,000 RPM for 60 minutes. The 161 gram weight was used because it was available, not because of any specific compaction force.

DI Water was added to the dry, compacted sediment in various increments using trialand-error to determine how much water the sediment would absorb and distribute. Based on the visible hydration experiments, a quantity of 10 grams of DI water could be added to the clay-coated quartz sediment and be absorbed within a period of 2-3 days.

An amount of DI water in excess of 10 grams would not be fully absorbed into the clay coating of the sediment, and would pool in the lowest aspect of the tube (as free water 
driven by gravity). Based on these results, a quantity of 10 grams of DI water was established as the optimum amount to be used for hydration of the artificial sediment.

A total of approximately 20 grams of DI water could be added to the artificial sediment before the excess water would fill the free volume of the pore space in the sediment.

After choosing the optimum amount of water to hydrate the kaolinite/quartz sediment, a comparison experiment was conducted using the ground quartz/quartz sediment. In a separate polycarbonate tube, 60 grams of dry ground quartz/quartz sediment was loaded and centrifuged identically to the kaolinite/quartz sediment. After compaction, 10 grams of DI water was added to this sediment to visually observe what happened to the water.

In Figure 17, the tube on the left contained kaolinite/quartz sediment that was wetted with 10 grams of DI Water. The tube on the right contained fine quartz/quartz sediment that was wetted with 10 grams of DI water. The DI water has been fully absorbed and distributed in the kaolinite/quartz sediment. The DI water has distributed somewhat in the fine quartz/quartz sediment, but gravity has caused some of the DI water to pool at the base of the tube.

A packing weight was used to slightly compact the sediment. A rubber stopper was used to prevent the DI water from evaporating. Figure 17 was taken 72 hours after the DI water was added to the artificial sediment. 


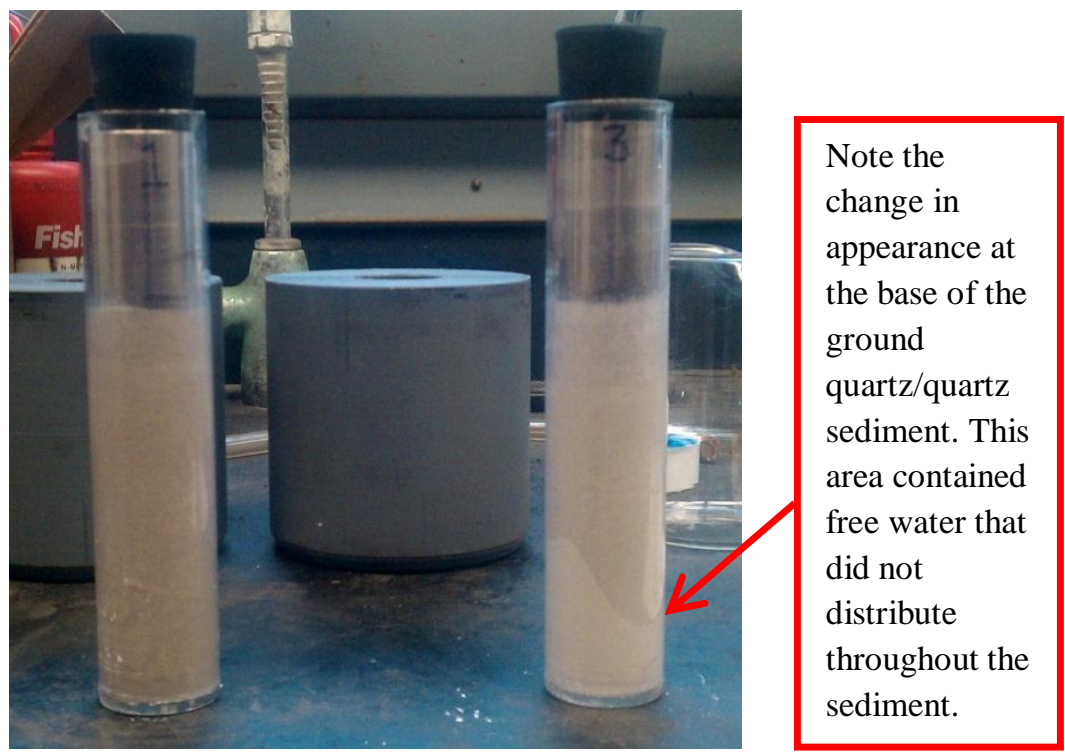

Figure 17: Wetted artificial sediments in polycarbonate tubes

\subsection{Computed Tomography (CT) Scanning of Artificial Sediment Mixtures}

Facilities exist at NETL for Computed Tomography (CT) scanning of sediment cores during methane hydrate formation experiments. The CT scanner used was a Universal HD 350E unit capable of scanning 1 millimeter slices of a sediment core ${ }^{19}$. The resolution of the Universal HD 350E scanner was $0.25 \mathrm{~mm}$ by $0.25 \mathrm{~mm}$ by $1 \mathrm{~mm}$. CT scans were performed for all experiments at $140 \mathrm{kV}$ and $100 \mathrm{ma}$.

In order to complete the experimentation, a cooling jacket was constructed for holding the APV. The cooling jacket was made of polyvinyl chloride (PVC), a material that is transparent to the X-Rays generated by the CT scanner. The cooling jacket, with sediment-filled APV, moved as the CT scanned 1-mm long circular slices of the sediment core. The total length of the sediment core was approximately $65-70 \mathrm{~mm}$ long. This resulted in 65-70 slices per sediment core. 
Images from the CT scanner were manipulated in ImageJ Software ${ }^{20}$ - freeware available from the National Institutes of Health. The manipulated images showed how methane hydrate formed in the sediment cores and whether the formation was uniform or not.

Variables monitored during the methane hydrate Formation Experiment were:

- Temperature of the cooling fluid and/or sediment core

- Inlet pressure at the top of the APV

- Exit pressure at the bottom of the APV

By combining the numerical data above with the image date generated by the CT scanner, a high resolution picture of what happened in the sediment core was seen.

\subsection{Experimental Apparatus for Methane Hydrate Formation Experiments}

A Piping \& Instrumentation Diagram (P\&ID) of the experimental set-up is shown in Figure 18. Images of the experimental apparatus are shown in Figures 19 through 22. 


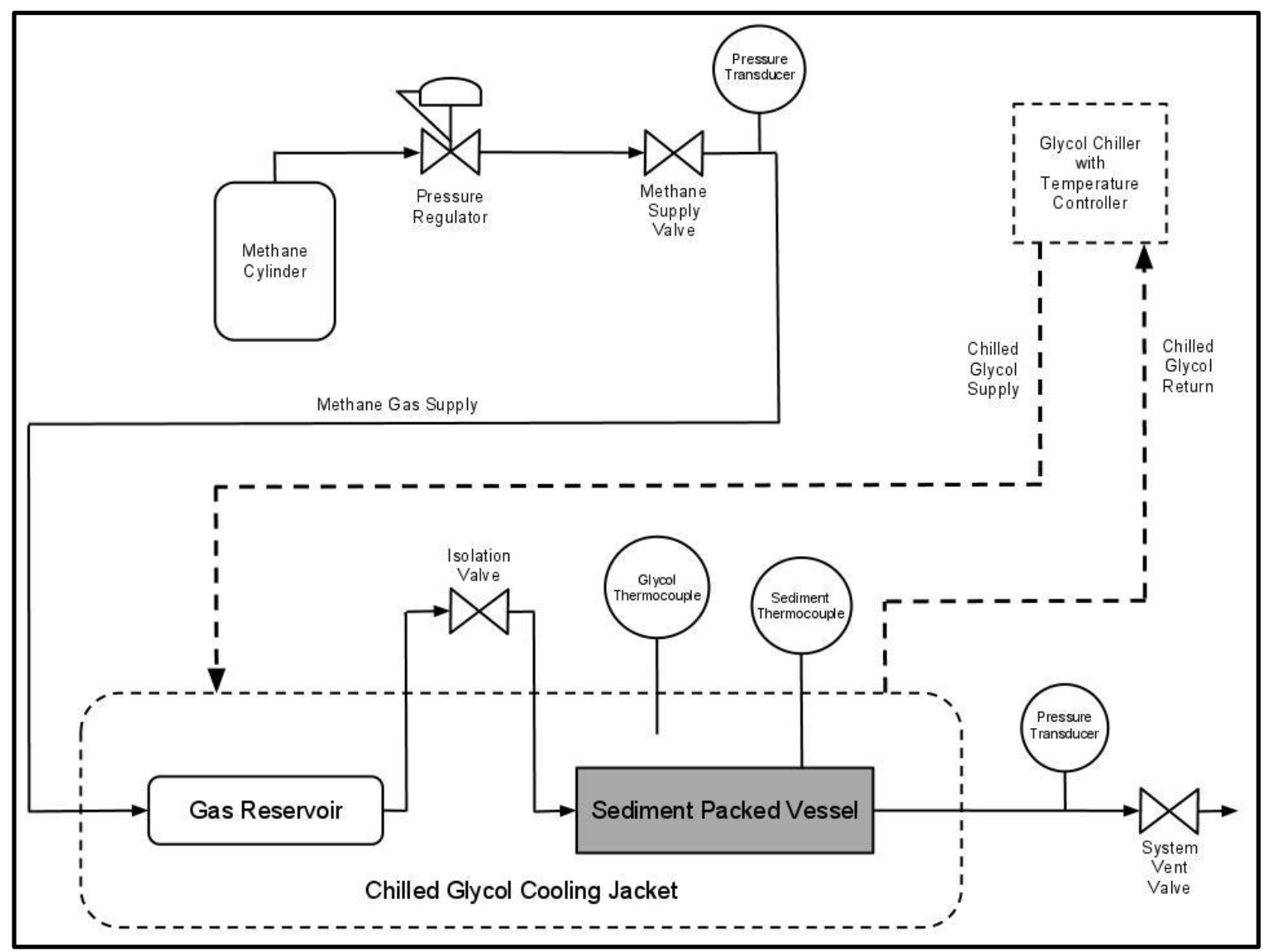

Figure 18: Methane hydrate experimental formation apparatus P\&ID 


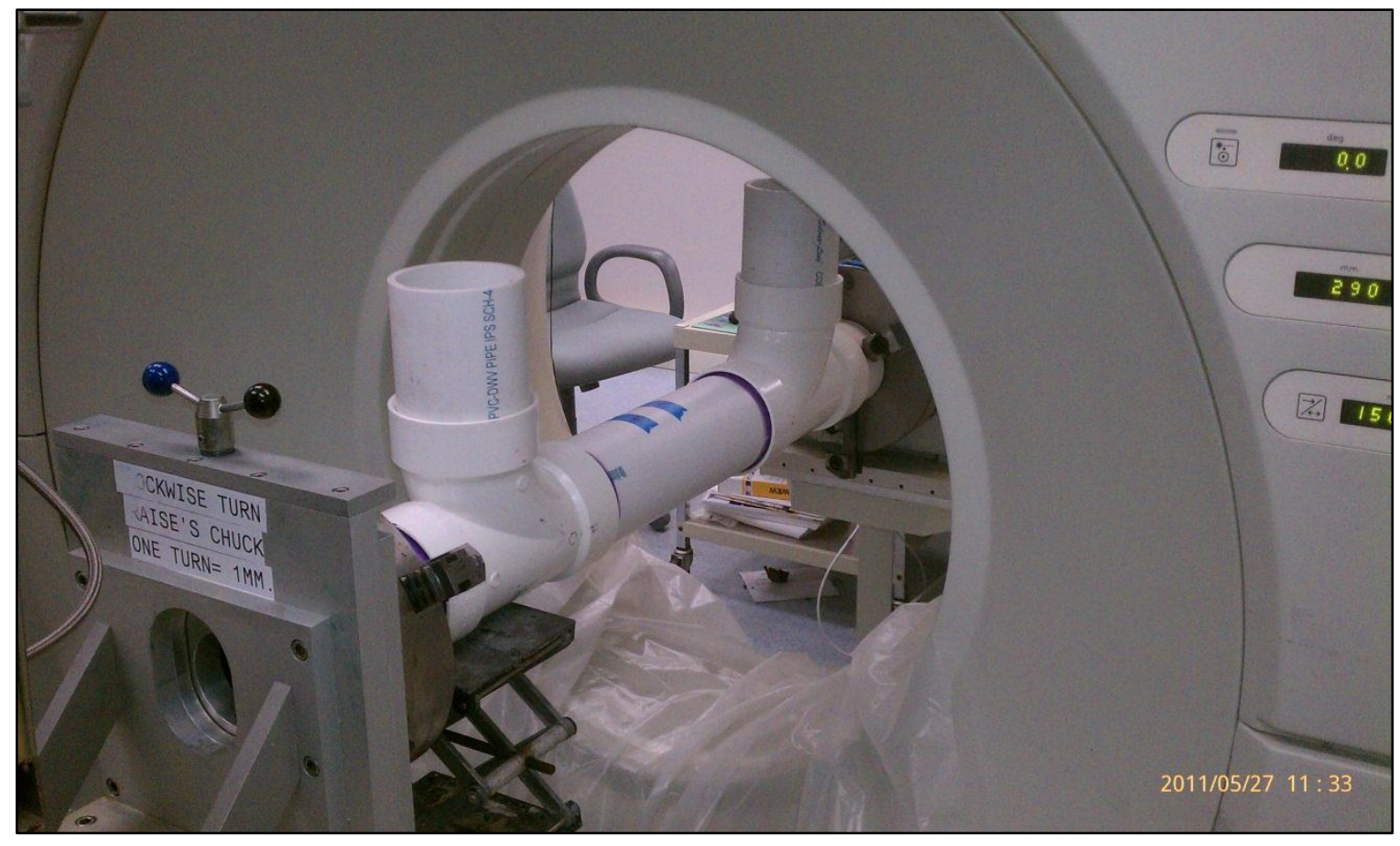

Figure 19: Glycol cooling jacket in CT scanner

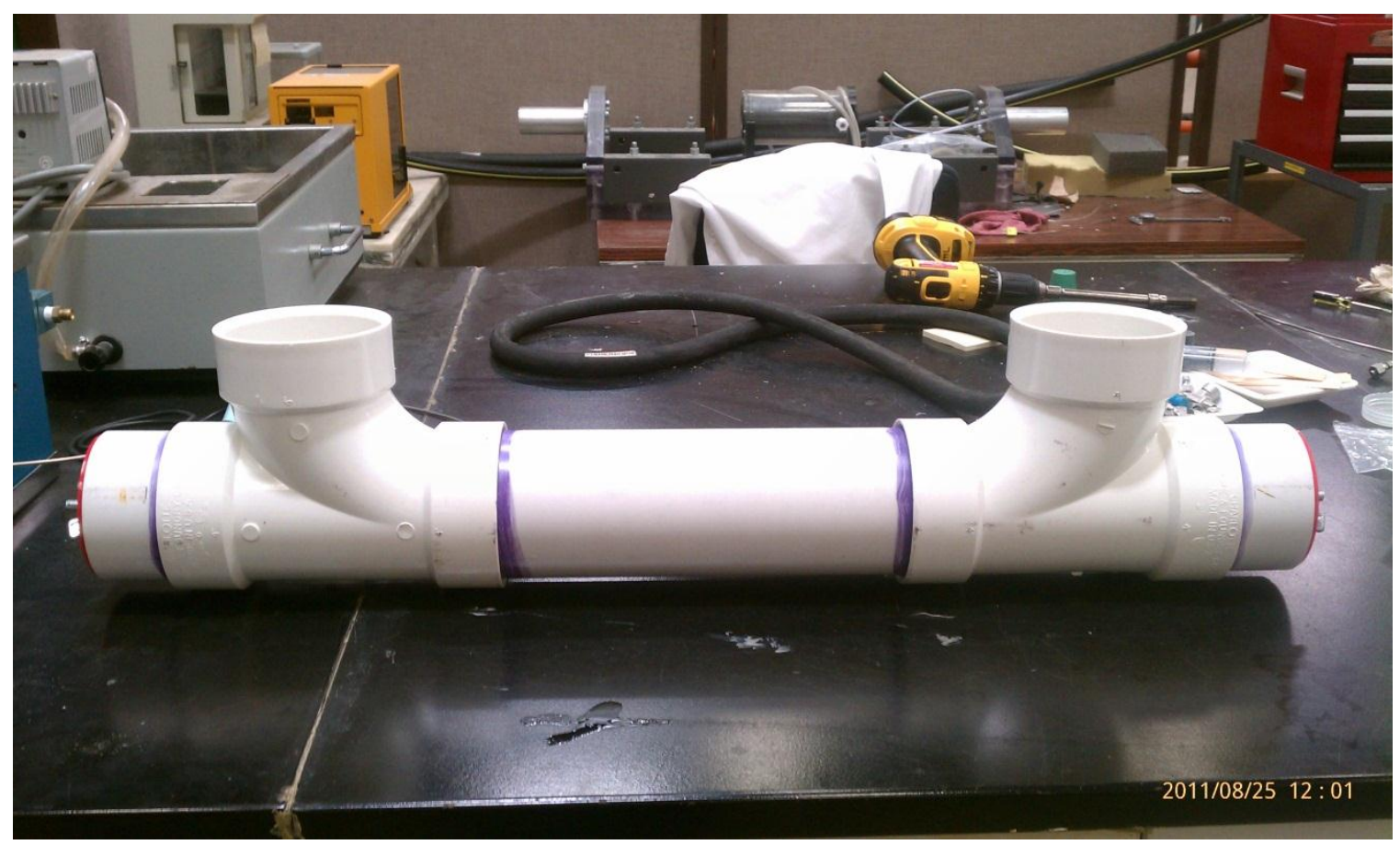

Figure 20: Glycol cooling jacket 


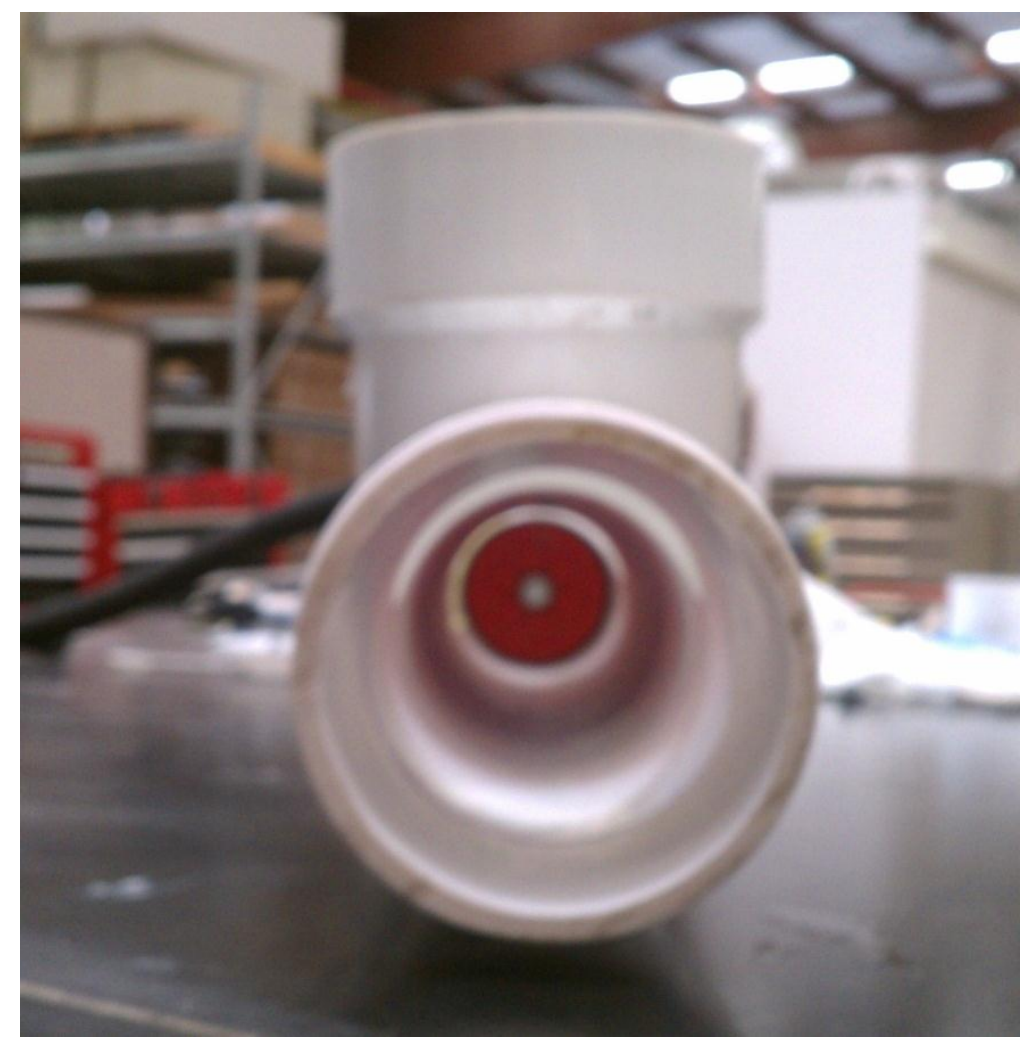

Figure 21: Interior view of glycol cooling jacket

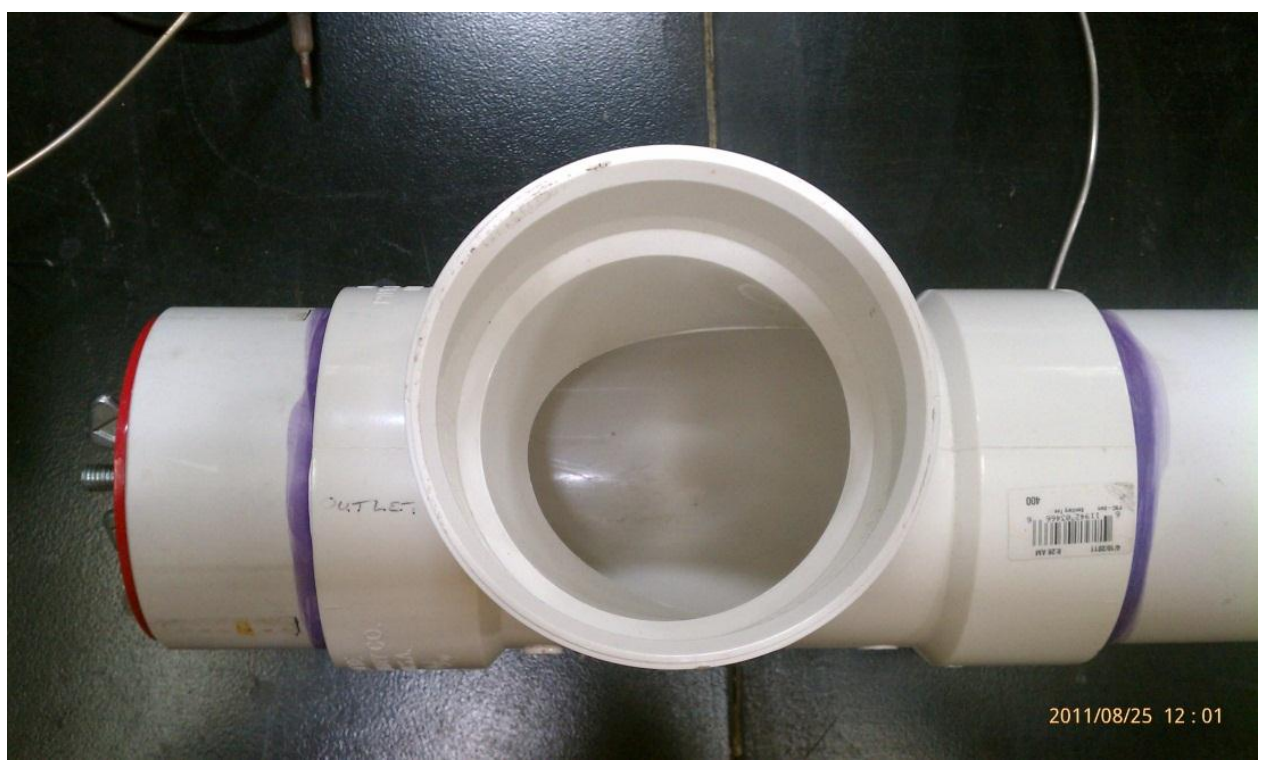

Figure 22: View into top opening of glycol cooling jacket 


\subsection{Experimental Procedure to Test Primary Hypothesis}

For each of the three experiments conducted, the experimental procedure was as follows:

1. Sediment samples were weighed, loaded into APV's, compacted, and hydrated at the Carbon Products Laboratory at WVU. APV's were transported to the NETL CT Scanning Facilities and placed into the Experimental Apparatus.

2. The sediment cores were flushed with methane to remove any air from the system.

3. The experimental apparatus was cooled using chilled glycol to the desired temperature, approximately $2-4^{\circ} \mathrm{C}$.

4. The system was pressurized with methane to approximately $1,100 \mathrm{psig}$. This pressure is typical for methane hydrate formation experiments conducted at NETL, and has been recommended by Dr. Yongkoo Seol.

5. Temperatures were measured with Type $\mathrm{K}$ thermocouples. Pressures were measured with pressure transducers. Temperature and pressure data were transmitted to a laptop and captured with Labview ${ }^{21}$ software. Labview saved the data to an Excel spreadsheet for future analysis.

6. At appropriate intervals, CT Scans were conducted of the experimental apparatus with a resolution of $0.25 \mathrm{~mm} \times 0.25 \mathrm{~mm} \times 1 \mathrm{~mm}$.

7. Each experiment was allowed to run until steady state pressure conditions existed. That is, when no change in pressure was detected for more than 1 hour, the experiment was terminated.

8. After steady state pressure was reached, the hydrate was dissociated by 
depressurization. Depressurization occurred by opening the system vent valve.

Thermal stimulation of the hydrate core was tried on the first sample, but this proved to be difficult to control. The equipment in the system was protected by a rupture disk with a rating of 2,000 psig. In order for the system pressure to not exceed the pressure rating of the rupture disk, a continuous vent path had to be open. When this was done, the system depressurized immediately. CT scans during depressurization proved to be impossible as the depressurization only took 30-60 seconds.

\section{$7 \quad$ Discussion}

Two artificial sediment samples were prepared for experimentation as detailed in the section "Preparation of Artificial Sediment Samples." The first sample was comprised of 90\% F110 sand mixed with 10\% kaolinite. The second sample consisted of 90\% F110 sand mixed with $10 \%$ quartz fines. Both samples were loaded into APV's and were centrifuged according to the sample preparation procedure.

After centrifugation, both samples had 10 grams of DI water added and the APV's were sealed to prevent evaporation. The water was allowed to disperse into the samples for 3 days before further work was done.

Both samples were taken to the NETL CT Scanning facilities. The F110 sand/kaolinite sample was the first to be used in experimentation. The system was assembled according

to the P\&ID shown in Figure 18. Each experiment was allowed to progress to steady state pressure conditions. Pressure and temperature data were captured during the entire experiment. 
The false-color images and pressure/temperature traces from each experiment are shown below. A discussion of each experiment is also shown below.

\subsection{Experiment \#1: 90\% F110 Sand with 10\% kaolinite and 10 grams of water}

Experiment \#1 began on June 7, 2011 at 14:42. The system was pressurized to 1,117 psi initially and an initial CT scan was conducted. The system remained pressurized throughout the night and into the following day. By 09:11 on June 8 , 2011, steady state pressure had existed for several hours at approximately $950 \mathrm{psi}$.

At this time, the valve from the gas reservoir was opened to the sediment core to determine if anything would happen. The pressure in the reservoir dropped approximately $100 \mathrm{psi}$, from $1,117 \mathrm{psi}$ to $1,018 \mathrm{psi}$. The exit pressure essentially remained the same (it was expected to rise). It was theorized that the core had a solid blockage due to hydrate formation. The experiment was allowed to remain under these conditions until 16:07. No change was detected. A final CT scan was taken prior to dissociation. The system was vented and depressurized.

The initial scan and final scan were manipulated in ImageJ software to generate an image of the hydrate formed. Figure 24 shows the image which was created by subtracting the individual slices of the wet sediment core from the final hydrate core. This image manipulation is a standard practice at NETL to show where differences occur in before and after images. In Figure 24, low density hydrate is blue, medium density hydrate is red, and high density hydrate is white. These densities are relative to this experimental

apparatus. Attempts were made to calculate absolute densities of the formed hydrate, but 
that proved impossible due to the variety of materials that X-rays had to pass through (air, PVC, glycol coolant, aluminum, sediment, water, and hydrate).

It can be seen that the hydrate has formed uniformly throughout the core. In Row 1, Columns 1 through 8, and Row 2, Columns 1 through 5 (which would have been at the bottom of the core), a certain amount of non-uniformity can be seen as a void in the color. This is probably due to a migration of fines during centrifugation and hydration of the core.

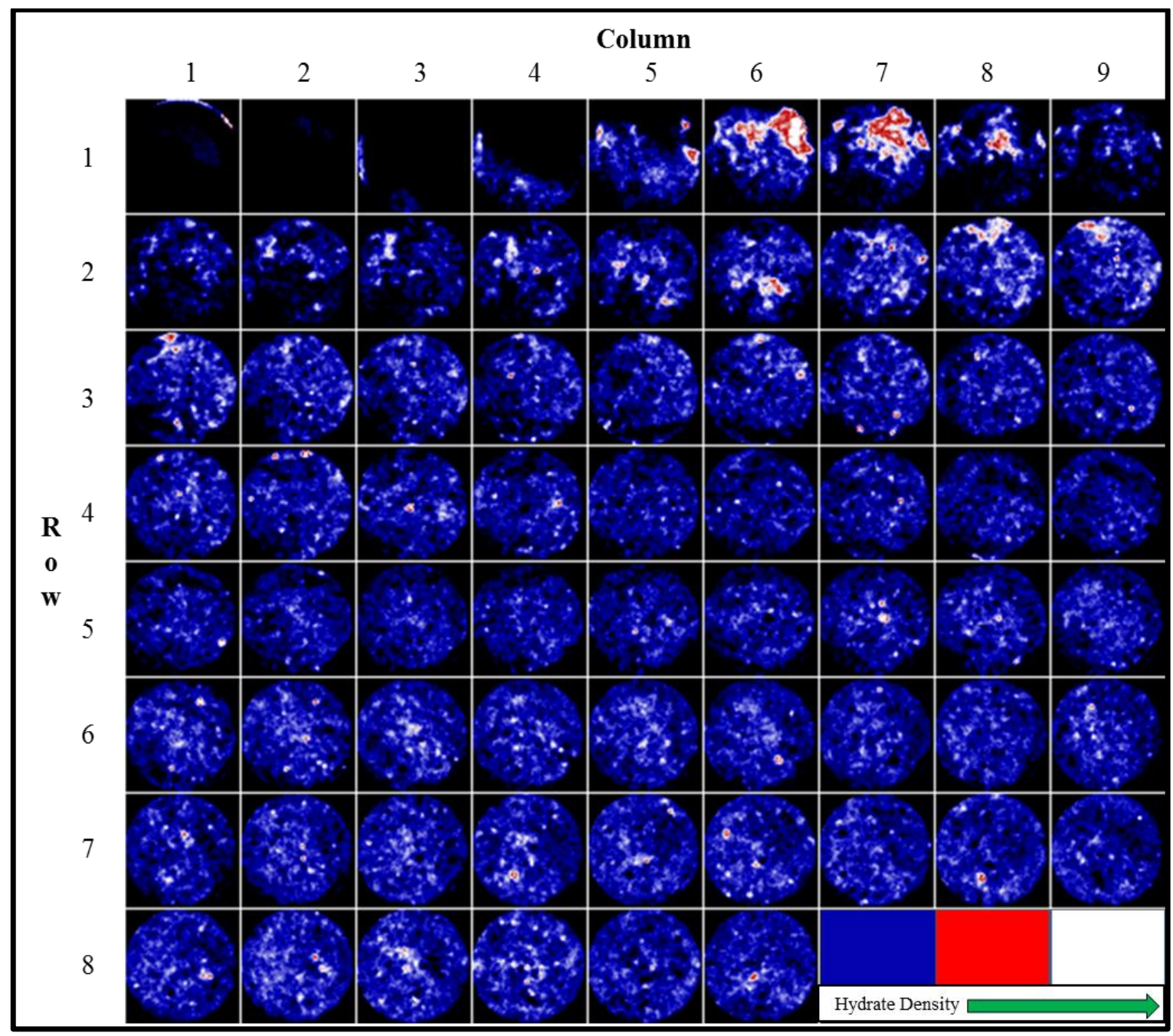

Figure 23: Experiment \#1 false-color image of hydrate-wet CT scans 
As a comparison, a similar image in Figure 24 from Seol and Kneafsey ${ }^{10}$ shows how nonuniform previous hydrate formation experiments could be.

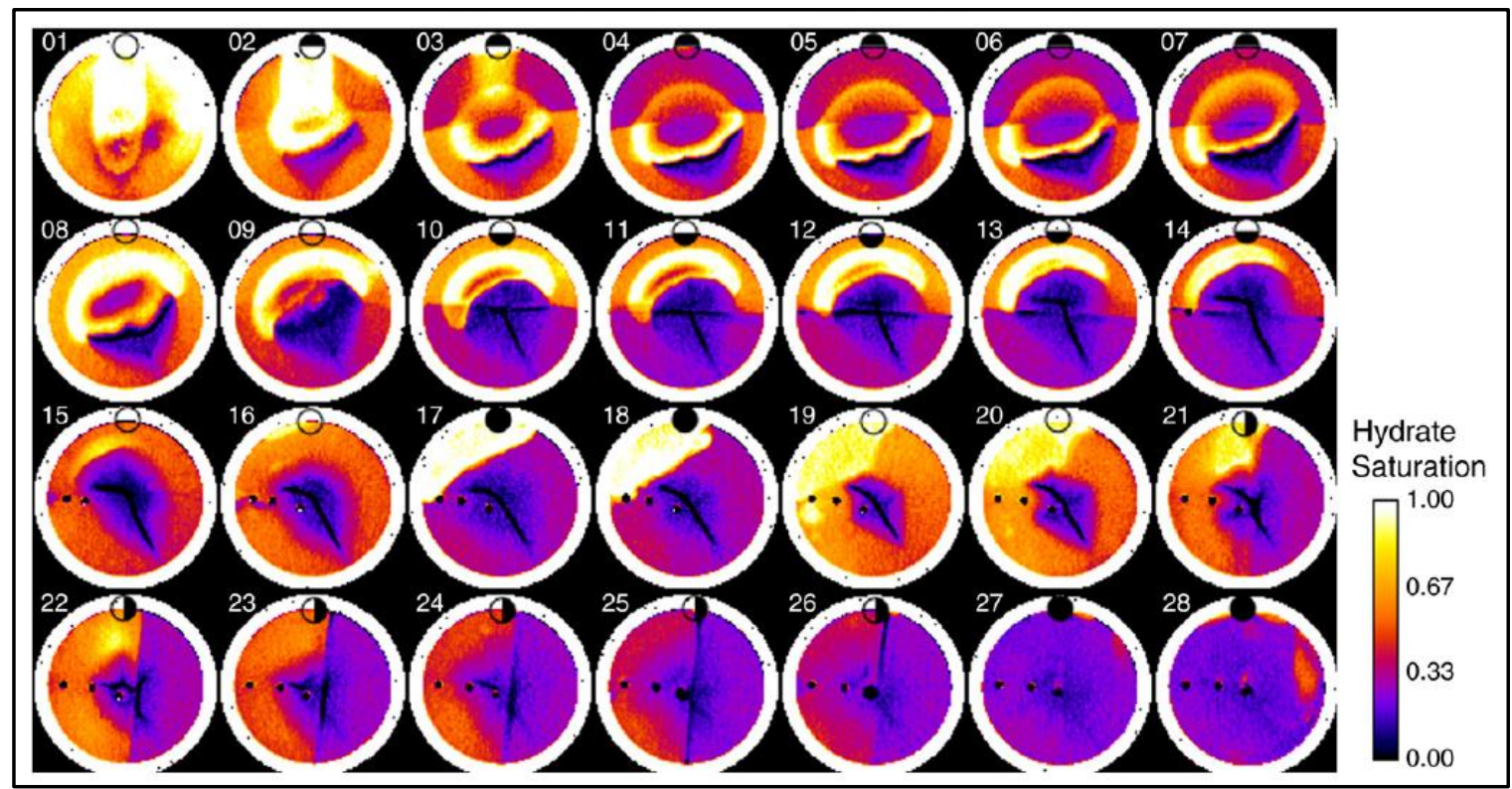

Figure 24: CT images showing hydrate saturation after the completion of hydrate formation. Circles on top of each image represent layer configuration, with open area for fine sand and filled area for coarse sand. A brighter elliptical artifact in the first three images was caused by accidental air injection during one of the later steps of the measurement sequence - from Seol \& Kneafsey ${ }^{10}$ experiment

Only one thermocouple was used in Experiment \#1. This measured the circulating glycol bath temperature. From later experiments, it was confirmed that there was virtually no difference between the glycol bath temperature and the sediment core temperature. Also, the gas reservoir was valved out throughout the entire experiment, which allowed only the methane initially in the APV to form hydrate.

The pressure and temperature traces are shown in Figures 25 and 26. The chilled glycol bath circulated at approximately 9 liters/minute (as measured with a bucket and a stopwatch), which cooled the experimental apparatus from ambient temperature to hydrate formation temperature in less than 10 minutes. This rate of cooling also rapidly removed the heat released from hydrate formation, which explained why no temperature 
rise was detected in the reservoir temperature.

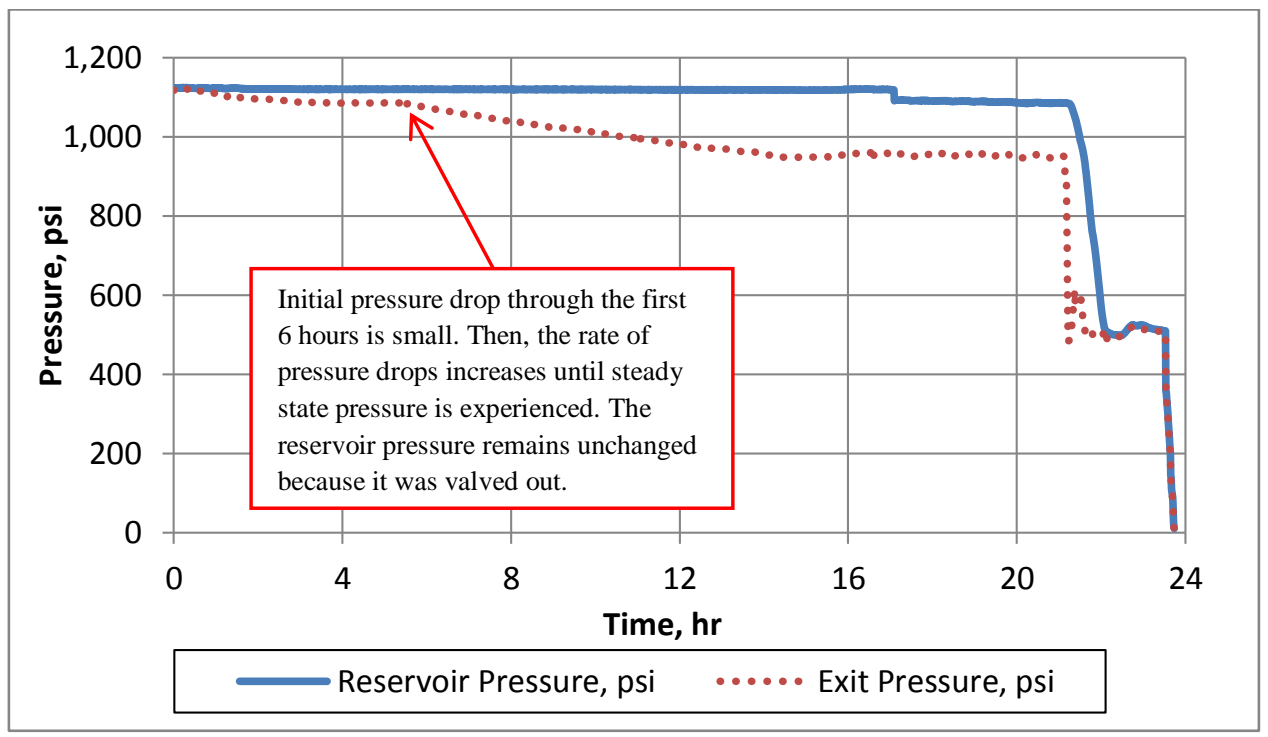

Figure 25: Reservoir \& Exit Pressure for Experiment \#1 - The exit pressure dropped slowly from Time 0 until 6 hours into the experiment. From 6 hours until 15 hours, the exit pressure drop was more rapid. After hour 15, the exit pressure did not change.

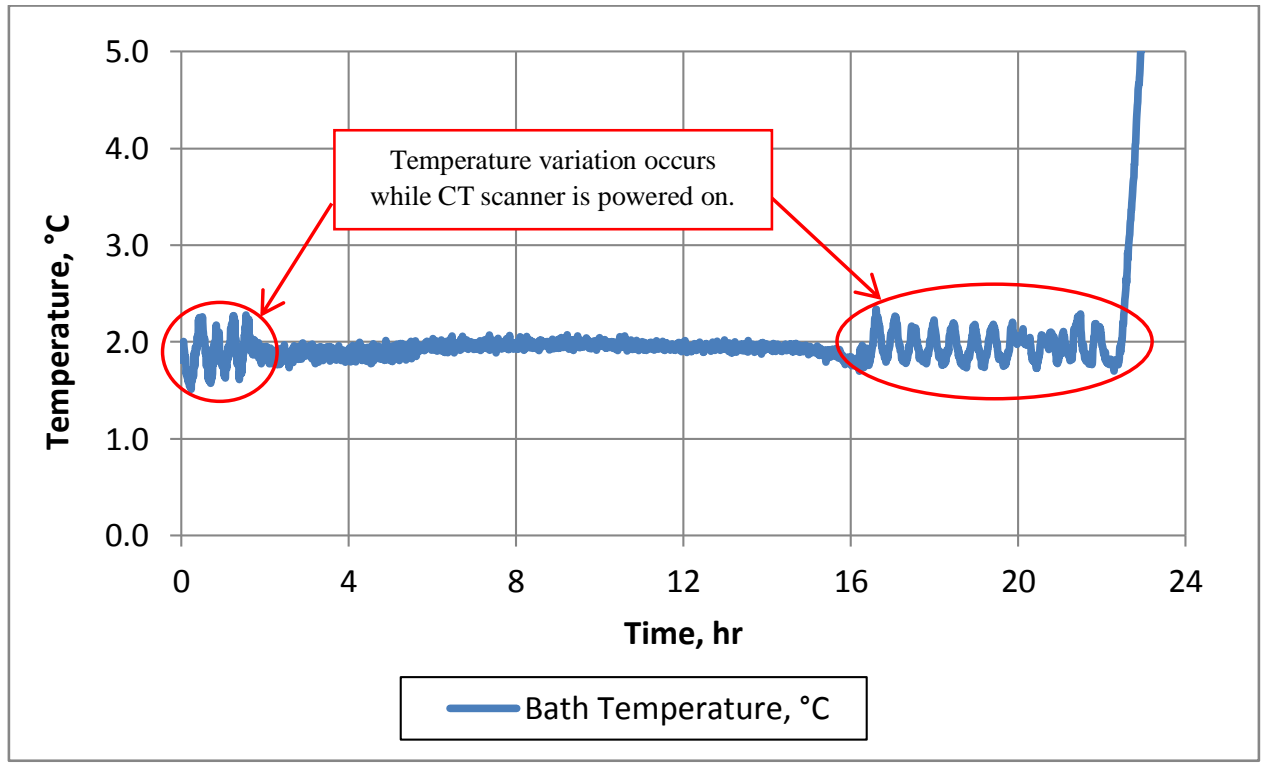

Figure 26: Glycol Bath Temperature for Experiment \#1 - The temperature showed little variation throughout the entire experiment. From Time 0 until hour 2 and from hour 16 to hour 22, a $0.25^{\circ} \mathrm{C}$ variation is due to the heat generated by the CT scanner. Overnight, the CT scanner was turned off. 


\subsection{Experiment \#2: $90 \%$ F110 Sand with 10\% kaolinite and 20 grams of water}

Upon completion of Experiment \#1, image analysis was conducted and reviewed with Dr. Yongkoo Seol. Upon Dr. Seol's recommendation, a variation of Experiment \#1 was conducted. The sediment core from Experiment \#1 was completely saturated with water. That is, water was dripped into the sediment sample while partially restricting the bottom discharge until a liquid level could be seen at the top of the sediment. The sediment sample was sealed in overnight to allow the water to absorb into the clay coating.

The rough estimate of additional water in the sediment sample was 5-10 grams. The weight is only an estimate, as the tubing fittings used in the saturation contained water when they were removed. There was also loose water in the small channels at the top and bottom of the sediment when it was re-assembled in the experimental apparatus. Some of this water may have been removed from the system when the sediment was purged with methane.

For Experiment \#2, the same procedure was used as in Experiment \#1. The system was pressurized to nearly $1,100 \mathrm{psi}$ and the glycol chiller was set to maintain $2^{\circ} \mathrm{C}$. Initially, the glycol temperature controller was providing a slightly cooler temperature than desired. Therefore, the glycol temperature controller was adjusted up by $1 / 2{ }^{\circ} \mathrm{C}$ several hours into the experiment.

Initial pressurization occurred on June 9, 2011 at 16:48. An initial CT scan was performed. At this time, the gas reservoir was valved out. Overnight, the pressure dropped quickly to less than $600 \mathrm{psi}$. The valve isolating the gas reservoir was opened on 
June 10, 2011 at 15:26. The gas reservoir and exit pressures equalized almost immediately.

On June 11, 2011 at approximately 03:00, the pressures began to diverge. The gas reservoir pressure continued to drop, but the exit pressure held at approximately at 830 psi before dropping to 770 psi. The exit pressure held at 770 psi for 30 hours. On June 12, 2011 at 21:00, the pressures began to equalize. For the remainder of the experiment, the pressures stayed nearly equal. A final CT scan was conducted on June 13, 2011 at 14:25. Then, the system was depressurized. Figure 27 shows the exit and reservoir pressures in graphical form.

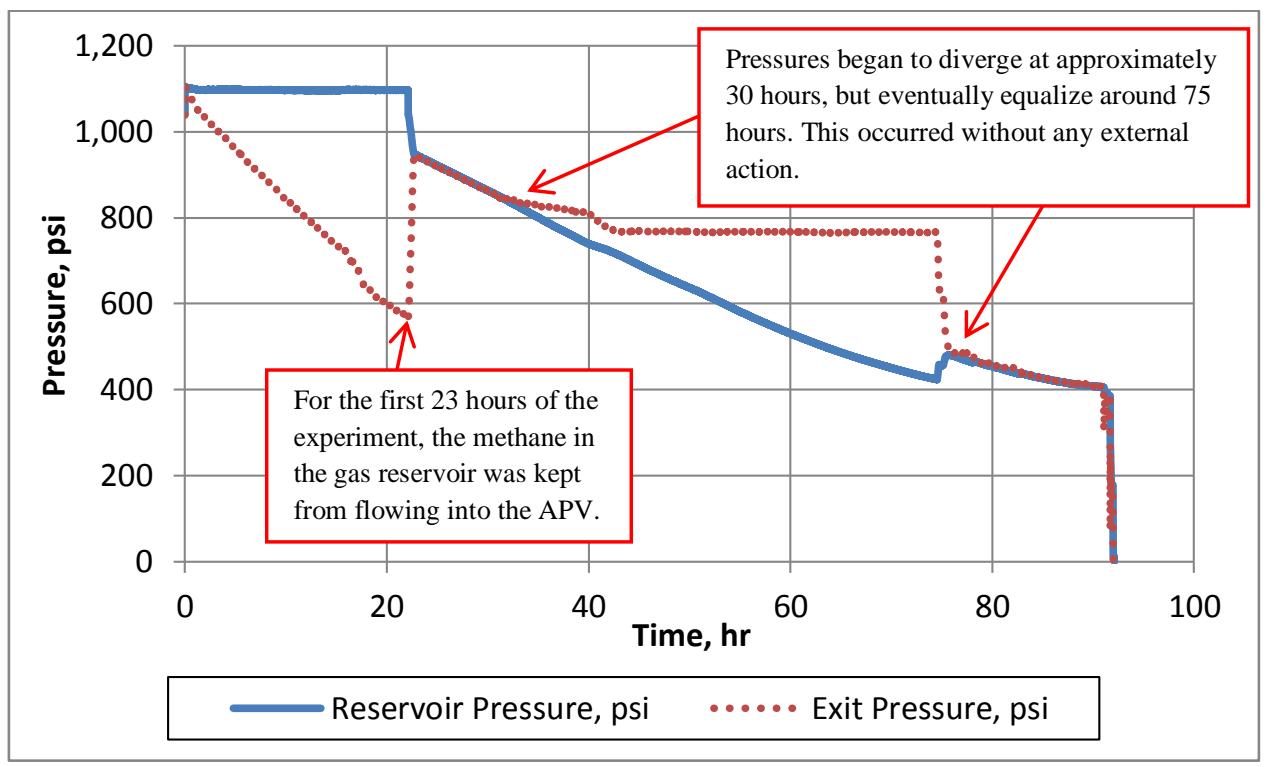

Figure 27: Reservoir \& Exit Pressure for Experiment \#2

A possible explanation for the pressure difference may be a blockage in the gas path through the sediment core from methane hydrate formation. The most likely spot for this to occur is at the base of the sediment, possibly in the APV exit channel, as this channel 
is narrow (approximately $1 \mathrm{~mm}$ in diameter) and may be a place for free water to accumulate.

This experiment took nearly 4 days to reach steady state pressure. The final pressure was $380 \mathrm{psi}$, which is roughly the equilibrium pressure for methane hydrate at $2^{\circ} \mathrm{C}^{22}$. Therefore, the data suggests that this experiment was gas limited.

Two thermocouples were used in this experiment. As before, the glycol bath temperature was measured. Additionally, a thermocouple was placed in the sediment core. The measuring tip was placed as close to the center as possible. There was almost no difference in temperature between the two thermocouples, and both responded to variations at the same time. The best explanation for this phenomenon is the combination of the APV and the wet sediment had high thermal conductivity.

Figure 28 shows the bath and core temperatures for Experiment \#2. An initial adjustment in the bath temperature was made to keep the bath at nearly the same temperature as Experiment \#1. Two areas of note exist in Figure 28. At 23 hours, a temperature spike is detected when the valve between the gas reservoir and APV was opened. The core quickly returned to its previous temperature, which showed the high rate of heat transfer in the APV. The second area of interest was the time from hour 40 until hour 87 . This time occurred on the weekend, and the CT scanner was turned off. Therefore, little variation was experienced in the bath and core temperatures. 


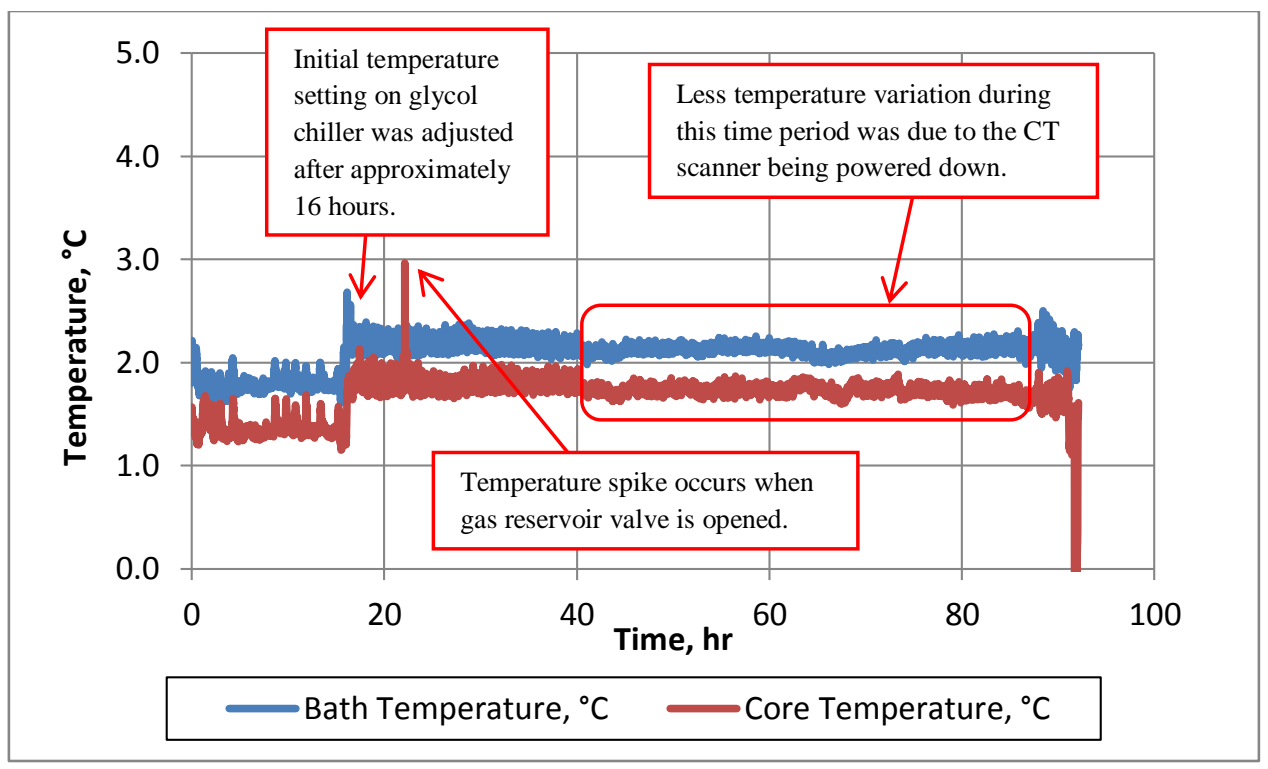

Figure 28: Glycol Bath and Sediment Core Temperatures for Experiment \#2

The ImageJ analysis in Figure 29 of the CT Scans showed another highly uniform methane hydrate formation. In Row 1, Columns 1- 9, and Row 2, Columns 1-7, some irregularities were seen at the bottom of the sediment. Beginning in Row 3, Column 7, the insertion of the thermocouple was seen throughout the rest of the images. Near the top of the sediment, in Row 8, Columns 1 through 6, more irregularities were seen. Having explained a likely scenario for the bottom of the sediment, the other two areas can also be explained.

By forcing the thermocouple into the sediment, this created loose sediment surrounding the thermocouple. This loose sediment does not form methane hydrate equally to the packed sediment lying further away. The top of the sediment was disturbed by the removal of the sintered metal plate during the extra water addition. The disturbed sediment was not nearly as compacted as the sediment further down the sediment core. 


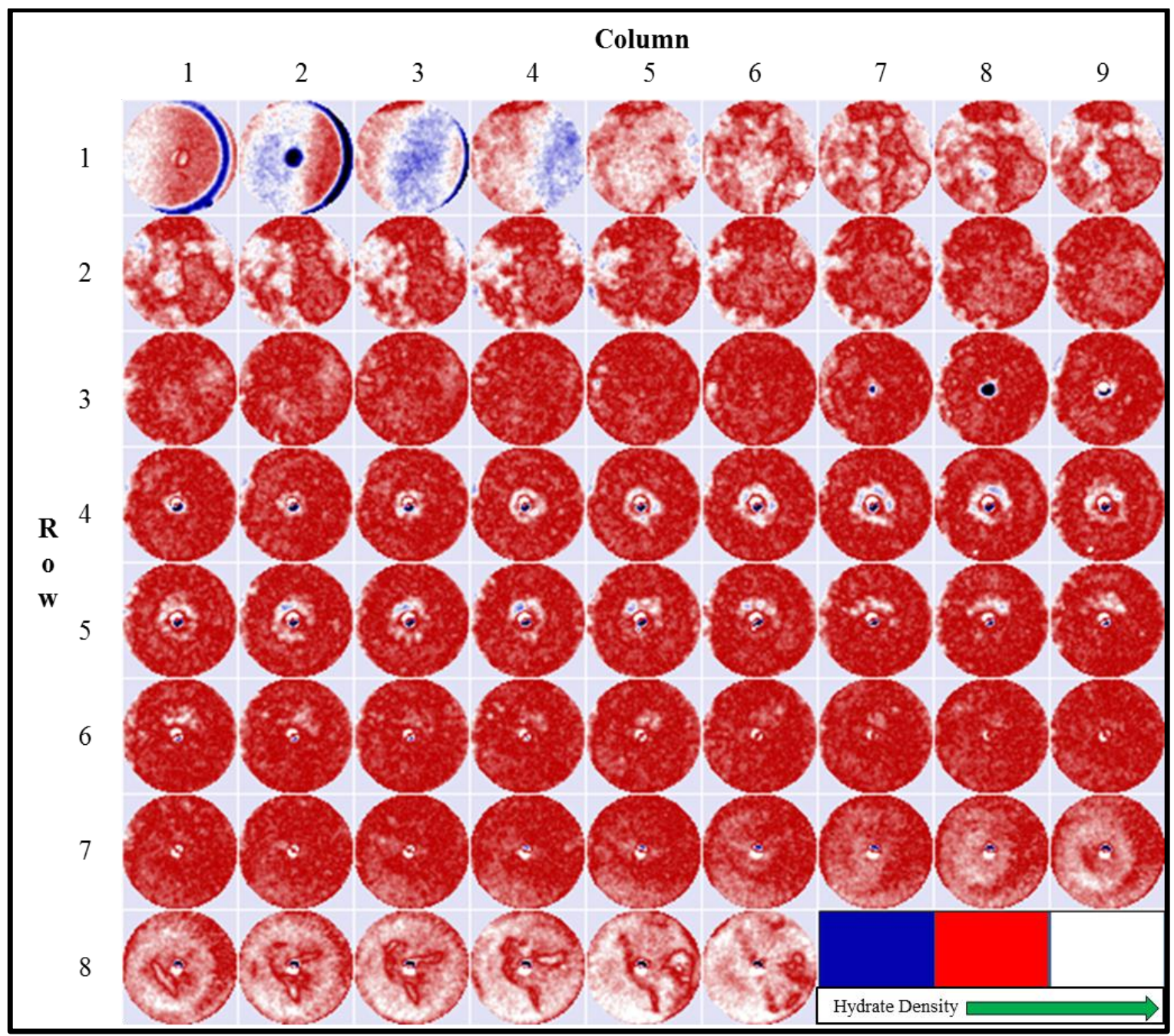

Figure 29: Experiment \#2 False-color Image of Hydrate-Wet CT scans - most of the hydrate formed in this experiment is of relative medium density. Some irregularities can be seen, but the bulk of the hydrate is uniform.

\subsection{Experiment \#3: $90 \%$ F110 Sand with 10\% quartz fines and 20 grams of}

\section{water}

Upon completion of Experiment \#2, further consultation with Dr. Seol led to Experiment \#3 being the analog of Experiment \#2. In this experiment, the F110 sand and quartz fines sediment was used, and was saturated with water before being placed in the experimental apparatus. For Experiment \#3, the same procedure was used as in the previous 
experiments. The system was pressurized to nearly 1,100 psi and the glycol chiller was set to maintain $2^{\circ} \mathrm{C}$.

Initial pressurization occurred on June 14, 2011 at 07:48. An initial CT scan was performed. Based on information from the previous experiment, the valve between the gas reservoir and the sediment was left open throughout Experiment \#3. As in Experiment \#2, the pressures diverged at times during the experiment, but did eventually recover to nearly the same value. On June 16, 2011 at 02:16, equilibrium pressure was reached. A final CT scan was performed, and the system was depressurized.

Experiment \#3 ran for slightly more than 2 days before reaching equilibrium pressure. The final pressure reached was $400 \mathrm{psi}$. Once again, this is roughly the equilibrium pressure for methane hydrate at $2^{\circ} \mathrm{C}$. As in Experiment \#2, the data suggests that this experiment was gas limited. The reservoir and exit pressure traces are shown in Figure 30.

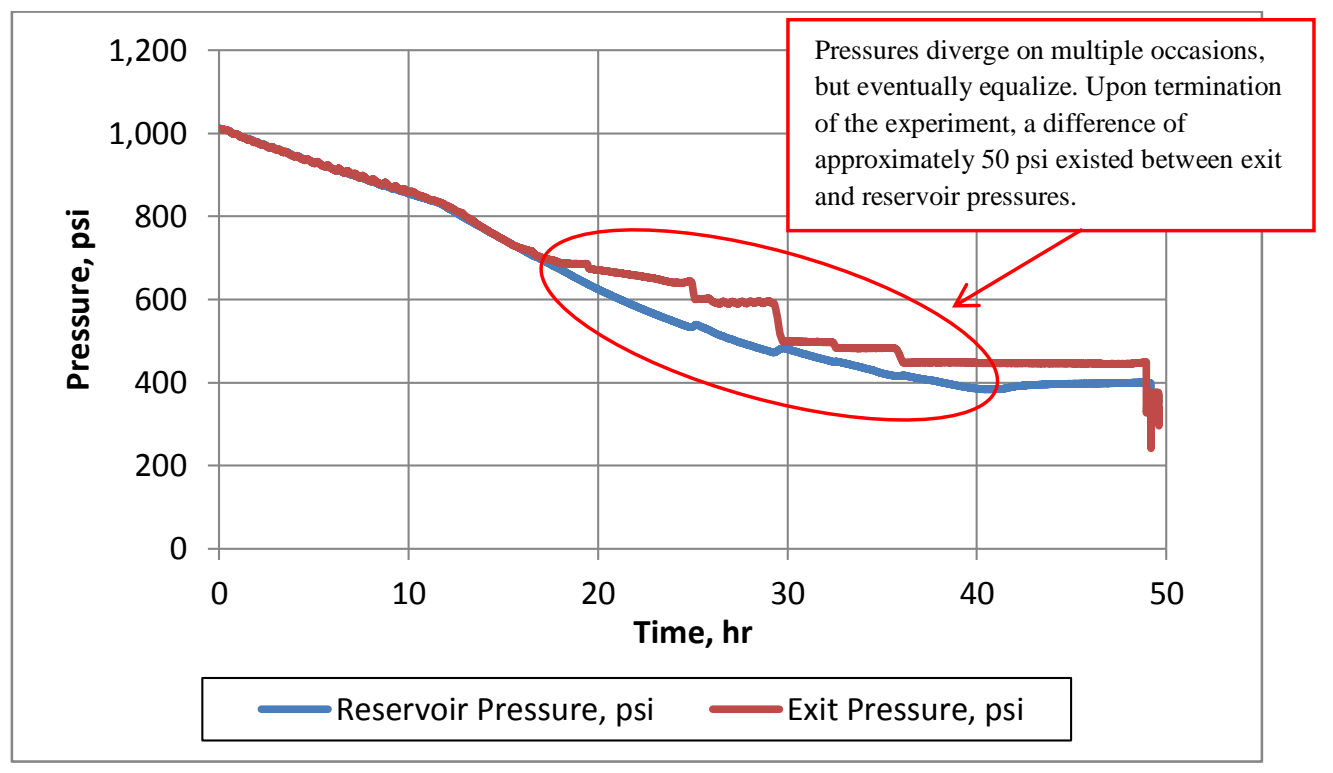

Figure 30: Reservoir \& Exit Pressure for Experiment \#3 
Two thermocouples were used in Experiment \#3, glycol bath and sediment core temperatures. There was almost no difference in temperature between the two thermocouples, and both responded to variations at the same time. Figure 31 shows the bath and core temperatures for Experiment \#3.

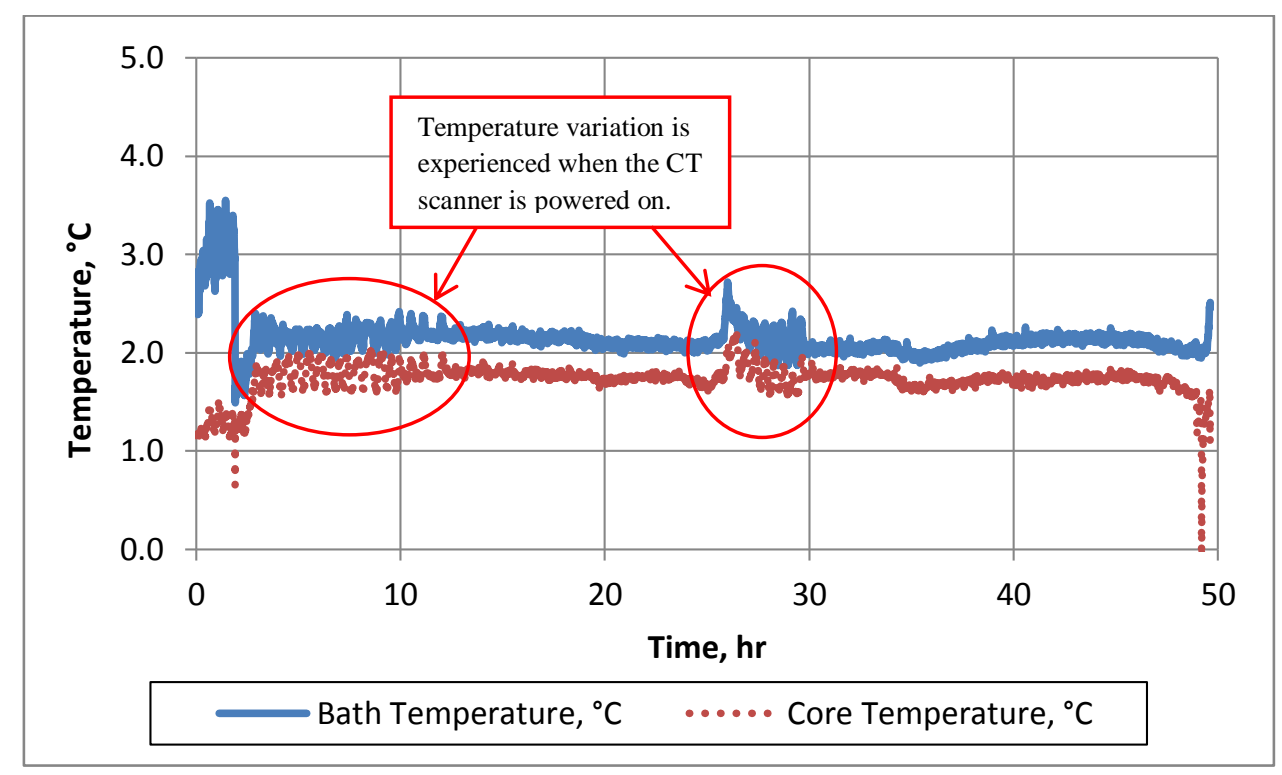

Figure 31: Glycol Bath and Sediment Core Temperatures for Experiment \#3

The ImageJ analysis of the CT scans in Figure 32 shows a very irregular methane hydrate formation. Areas of mostly low and medium density hydrate were formed in the Experiment \#3 sediment, with small areas of high density hydrate. The areas of greatest density were formed at either end of the APV as shown in Rows 1, 2, 7, and 8. The lowest density hydrate was formed in the middle of the APV in Rows 4, 5, and 6. 


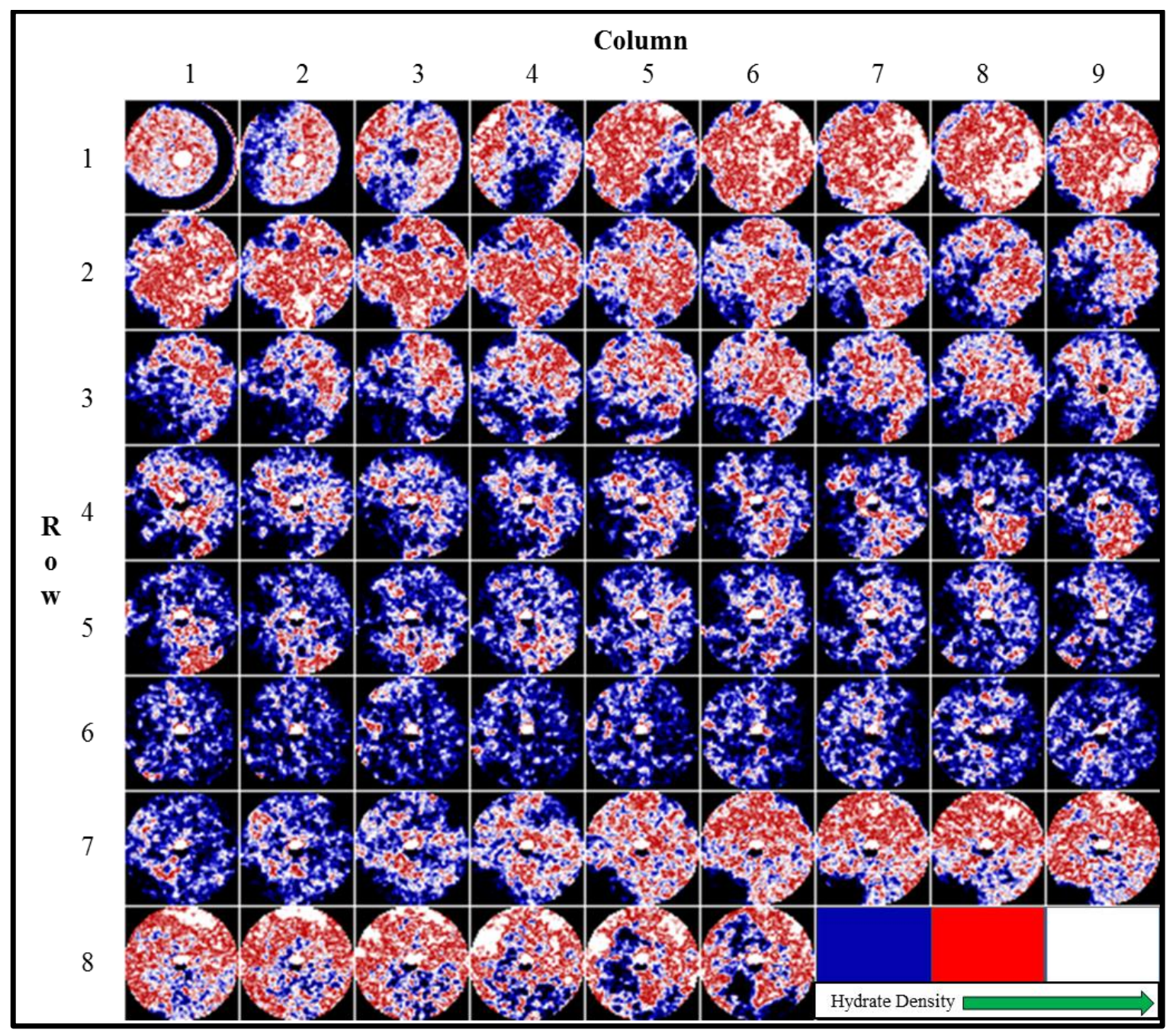

Figure 32: Experiment \#3 False-color Image of Hydrate-Wet CT Scans

Another point of interest in these experiments is the rate at which methane hydrate forms.

As noted in the Discussion section, the time that Experiment \#2 and Experiment \#3 took

to reach equilibrium were significantly different. Experiment \#2 (with kaolinite) took approximately 92 hours to reach equilibrium. Experiment \#3 (without kaolinite) took only 42 hours to reach equilibrium. This is illustrated in Figure 33. 


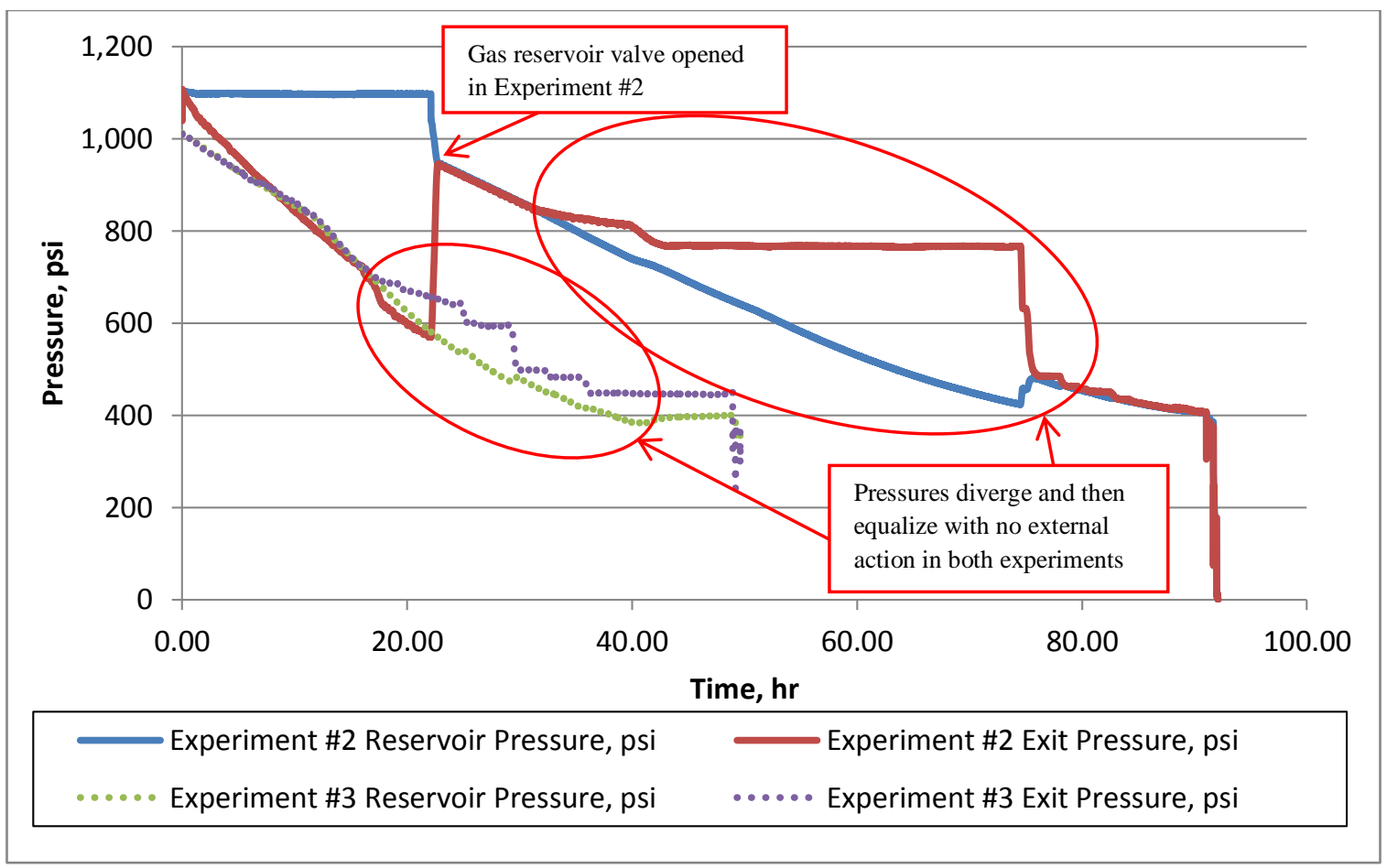

Figure 33: Reservoir and Exit Pressures for Experiments \#2 and \#3 


\section{Conclusions}

The data presented show that methane hydrate can be formed in sediment that does or does not contain clay minerals. However, when kaolinite is present in the sediment, water and methane remain distributed throughout the sediment. This distribution then drove a uniform methane hydrate formation when the proper conditions existed. This conclusion was postulated initially when viewing the visual packing experiments described in Section 6c.

The pressure traces show that kaolinite affected the formation rate of hydrate. The formation rates of methane hydrate appear to be different depending upon sediment composition. The presence of clay more than doubled the hydrate formation time.

It is not known what mechanism caused this phenomenon. A scenario could be postulated that the kaolinite coating slowed down the rate of diffusion of methane through the sediment during hydrate formation.

The data presented in this thesis supported the primary hypothesis that sediment composition played a role in both the uniformity and rate of methane hydrate formation. 


\section{$9 \quad$ Recommendations}

As with every experiment, after reviewing the data and information, some changes would be contemplated before future experimentation would be performed.

The first change would be to take the clay-coated dry sediment, and shake it lightly over a 100-micron screen. This would remove most of the loose clay particles. Care should be taken not to vigorously shake the screen, as this may scour some of the clay coating off the larger grain quartz. If a large diameter screen were used, this would prove to be the most effective, as a small depth of sediment would allow the fines to drop through the screen almost immediately.

Secondly, further experimentation can be conducted on various sediment compositions to determine if the rates of formation of hydrate differ. The fines content of each of the two sediments used were uniform, meaning that they were only quartz or only kaolinite. The composition of the fines can be varied to determine what effect, if any, this has on the rate of hydrate formation. CT scanning of this experimentation would not be necessary. Simple tracking of pressures and temperatures would provide the data needed.

Lastly, experimentation on mixed sediment cores may provide some interesting information. A sediment core could be prepared where one-half of the core was comprised of only quartz and the other half had a clay/quartz mixture. This experiment would need to be conducted in a CT scanner to view the resulting methane hydrate formation. 


\section{$\underline{\text { References }}$}

${ }^{1}$ Collett, T., Lee, M., Agena, W., Miller, J., Lewis, K., Zyrianova, M., Boswell, R., and Inks, T., Permafrost-associated natural gas hydrate occurrences on the Alaska North Slope, Marine and Petroleum Geology, Volume 28, Issue 2, February 2011, Pages 279-294

${ }^{2}$ Rose, K., Boswell, R., Collett, T., 2011. Mount Elbert Gas Hydrate Stratigraphic Test Well, Alaska North Slope: Coring operations, core sedimentology, and lithostratigraphy. Marine and Petroleum Geology 28 (2), 311-331.

${ }^{3}$ http://www.malvern.com/LabEng/products/Mastersizer/MS2000/mastersizer2000.htm

${ }^{4}$ http://www.inxitu.com/html/Terra.html

${ }^{5}$ http://www.materialsdata.com/

${ }^{6}$ http://pubs.usgs.gov/of/2003/of03-001/htmldocs/nomenclature.htm

${ }^{7}$ Stern, L., Kirby, S., Durham, W., Circone, S., and Waite, W., Laboratory synthesis of pure methane hydrate suitable for measurement of physical properties and decomposition behavior, Natural Gas Hydrate, in Oceanic and Permafrost Environments, M.D. Max, Ed. Kluwer Publications, 2000. 
${ }^{8}$ Turner, D., Cherry, R., and Sloan, E., Sensitivity of methane hydrate phase equilibria to sediment pore size, Fluid Phase Equilibria, 228-229 (2005), 505-510.

${ }^{9}$ Lu, H., Kawasaki, T., Ukita, T., Moudrakovski, I., Fujii, T., Noguchi, S., Shimada, T., Nakamizu, M., Ripmeester, J., and Ratcliffe, C., Particle size effect on the saturation of methane hydrate in sediments - Constrained from experimental results, Marine and Petroleum Geology (2005), 1-5.

${ }^{10}$ H. Ganji, M. Manteghian, and H. Rahimi Mofrad, Effect of mixed compounds on methane hydrate formation and dissociation rates and storage capacity, Fuel Processing Technology 88 (2007), 891-895.

${ }^{11}$ Reistenberg, D., West, O., Lee, S., McCallum, S., and Phelps, T., Sediment surface effects on methane hydrate formation and dissociation, Marine Geology 198 (2003), 181190.

${ }^{12}$ Xiaoya, Z., Jianwei, D., Deqing, L., Shuanshi, F., and Cuiping, T., Influence of A-type Zeolite on Methane Hydrate Formation, Chinese Journal of Chemical Engineering, 17(5), 854-859 (2009).

${ }^{13}$ http://www.britannica.com/EBchecked/topic/120723/clay-mineral

${ }^{14}$ http://www.britannica.com/EBchecked/topic/656561/zeolite 
${ }^{15}$ Zhang, P., Wu, Q., Jiang, G., Pu, Y., Relation between soil matrix potential changes and water conversion ratios during methane hydrate formation processes in loess, Journal of Natural Gas Chemistry 20(2011), 140-144.

${ }^{16}$ http://www.britannica.com/EBchecked/topic/346022/loess

${ }^{17}$ Seol, Y., and Kneafsey, T., X-ray computed-tomography observations of water flow through anisotropic methane hydrate-bearing sand, Journal of Petroleum Science and Engineering 66 (2009) 121-132.

${ }^{18}$ Young, H., Freedman, R., Sandin, T., Ford, A., Sears and Zemansky's University Physics (10th Edition), 2000.

${ }^{19}$ http://www.universal-systems.com/HD_350.php

${ }^{20}$ http://rsbweb.nih.gov/ij/

${ }^{21}$ http://www.ni.com/labview/

${ }^{22} \underline{\text { Sloan}}$, E., Koh, C., Clathrate Hydrates of Natural Gases, 2008. 


\section{Appendix 1: Particle Size Analysis Methodology}

This document was prepared for use at the NETL Methane Hydrate Laboratory and is used with permission from Ms. Kelly Rose. 


\section{Particle Size Analysis Methodology Using MasterSizer 2000}

\section{Device Description}

The device used for particle size analysis of core samples is a Malvern MasterSizer 2000 (MS2000) Laser Diffraction Particle Size Analyzer equipped with a Hydro 2000G liquid sample system. The MS2000 was connected to a tap water supply and drain for automatic operation. The MS 2000 was interfaced with a laptop PC which used standard Malvern software for running the device. The setup of the MS2000 was conducted by the factory authorized representative. No modifications were made to the device.

\section{Chemicals, Auxiliary Equipment and Consumables}

Additional auxiliary equipment and consumable were used in the analysis of the core samples. A list of these is below:

- Distilled water used to make solutions and core sample/water slurries

- Sodium Hexametaphosphate ( $\mathrm{SH}$ ) solution - chemical mixed with distilled water, which was used as a dispersant

- 50 milliliter VWR screw top sample tubes used for holding core sample/water slurries

- Fisher Scientific miniRoto stirring device to thoroughly mix core sample/water slurries

- Laboratory scale for weighing SH and samples

- Miscellaneous spatulas, water/solution bottles, markers, tape, sample bags, etc

\section{Sample Preparation}

A solution was prepared of 2.7 grams of sodium hexametaphosphate ( $\mathrm{SH}$ ) in 500 milliliters of distilled water for use as a dispersant solution for the core samples. The dispersant allows any agglomerated particles to separate for proper particle size analysis. Approximately $20 \mathrm{ml}$ of SH solution was placed in a $50 \mathrm{ml}$ sample tube along with a core sample, of a size approximately the end of a microspatula (or roughly 0.25 grams). The cap was screwed onto the sample vial and the slurry was spun using the Fisher Scientific miniRoto for approximately 20 seconds.

Initial testing of samples showed that the dispersant needed some amount of time to thoroughly disperse the core sample. It was determined that at least 30-60 minutes was needed, however most samples were left to sit overnight for proper dispersion. Samples were typically spun on the miniRoto at least 3 times before being placed into the MS2000 (after initial preparation, after 1 hour, and right before pouring into the Hydro 2000G).

Several trials were run on core sample concentration in the $\mathrm{SH}$ solution. It was a trial and error basis that determined that the best amount of core sample in the SH solution - an amount which allowed all $20 \mathrm{ml}$ of SH solution with slurried core sample to be poured into the Hydro $2000 \mathrm{G}$ and have the obscuration level within the manufacturer's recommended 13-20\% level. Vials which had higher concentrations of core sample showed variability in the analysis of different cuts due to coarse particles settling out in the few seconds it took to remove the cap from the sample vial after spinning. 


\section{MasterSizer 2000 Software Standard Operating Procedures}

The MS2000 uses a Standard Operating Procedure (SOP) to perform the analysis on each sample. Components of this SOP include: Stirrer speed, pump speed, ultrasound percentage, number of seconds of sonication applied prior to measuring particle size,

Conducting an Analysis

The same SOP was run for every sample. Upon starting the SOP, the MS2000 goes through a setup and check phase to ensure the laser and detectors are on-line. The MS2000 then prompts the operator to add sample to the Hydro 2000G.

Prior to adding the sample to the Hydro 2000G, the vial was spun on the miniRoto for approximately 20 seconds. This ensured a thorough mixing of the slurry. The cap was removed from the vial and the entire $20 \mathrm{ml}$ sample was poured into the Hydro 2000G. A small amount $(\sim 5 \mathrm{ml})$ of distilled water was then used to flush the vial, which was also poured into the Hydro 2000G. This rinsing was done to ensure that all course particles were included in the particle size analysis.

The operator then continues the SOP, which runs automatically. A series of three (3) analyses are run on each sample. The software displays each of the three (3) analyses, along with an average. This data is saved for further statistical analysis.

Occasionally, after the sample and distilled water were poured into the Hydro $2000 \mathrm{G}$ the obscuration percentage exceeded the desired maximum of $20 \%$. When this occurred, additional tap water was slowly poured into the Hydro $2000 \mathrm{G}$ to reduce the obscuration to the limits of $13-20 \%$. If a sample did not meet at least $10 \%$ obscuration, then an additional vial of that core sample was prepared and analyzed.

Upon completion of the SOP, three (3) automatic water clean cycles of the equipment were conducted by the MS2000 software. This ensured all residual material had been flushed from the equipment. Upon completion of sample analysis for the day, or at the beginning of a day, three (3) water cleaning cycles were conducted. 


\section{Appendix 2: X-Ray Diffraction Analyses Methodology}

This document was prepared for use in the NETL Methane Hydrate Laboratory and is used with permission from Ms. Kelly Rose. 


\section{$\underline{\text { XRD/XRF Analysis Methodology Using InXitu Terra Portable Device }}$}

\section{Device Description}

The device used for X-Ray Diffraction (XRD) and X-Ray Fluorescence (XRF) analysis of core samples is an InXitu Terra Portable XRD/XRF device connected wirelessly to a laptop computer. The InXitu Terra is a portable device capable of both XRD and XRF. The wireless interface transfers data to the laptop and data is viewed using Microsoft Internet Explorer or similar software. The InXitu Terra can be operated on an internal rechargeable battery or can be directly connected to 110 volt power via a power cord. No modifications are made to the device.

\section{Chemicals, Auxiliary Equipment and Consumables}

Additional auxiliary equipment was used in the analysis of the core samples. A list of the equipment is below:

- Electric oven for drying samples and warming grinding/sifting equipment

- PTFE sample dishes for holding samples while drying

- Mortar and pestle

- 125-150 Micron screen

- Miscellaneous spatulas, markers, tape, sample bags, etc

$\underline{\text { Sample Preparation and Analysis }}$

Approximately 5 gram of sample is removed from the original sample bag and placed on a PTFE dish and placed in the oven to dry. The ceramic mortar and pestle and brass screening trays are also placed in the oven to dry and keep warm. Sample drying times vary based on the water content and composition of the sample, but are normally 1-3 hours. After the sample is deemed to be dry enough for grinding, the sample is removed from the oven and placed in the clean/dry mortar and ground with the pestle until the nominal size is 150 micron or less. The InXitu Terra requires the sample to be 150 micron or less.

The ground sample is placed in the warm 150 micron screen to remove particles coarser than 150 microns. Some samples with coarser material are reground 1-2 more times to yield as much sample as possible. The second and third grinding steps are important, as the harder particles (such as zircon or pyrite) will be screened out during the first screening. This leaves a higher concentration of softer particles (such as clay) in the sample. The grinding and screening equipment should be kept warm to minimize the amount of moisture the clay can absorb from the air. The screened sample is then placed in a labeled sample bag.

Using a microspatula, a sample approximately the size of a pencil lead is removed and placed in the sample tray of the InXitu Terra. The sample is shaken using the external shaking mechanism to ensure the sample window is filled. The sample tray is then placed in the InXitu Terra and locked in place.

The Operating Software is maintained on the InXitu Terra and is used via the web interface. When the sample is in place, the analysis software is started. When the analysis is complete, the XRF image and the MDI-ka file are saved on the research LAN 
for viewing and analysis. The MDI-ka file is imported into Jade for mineral identification.

\section{$\underline{\text { InXitu Terra Operating Parameters }}$}

Several trials were run on core samples using different settings for the InXitu Terra. The basic set-up of the device exposed the sample to $250 \mathrm{x}$-ray exposure repeats each lasting 10 seconds. This number of exposures required an analysis time of approximately 60 minutes per sample. In order to determine the variability of the instrument, identical samples were run with a differing number of exposures. The numbers of exposures chosen for the trials were 10,100, and 1000. The 10 second setting per exposure was maintained.

At 10 exposures, the granularity of the XRD scan limited the identification of smaller peaks. At 100 exposures, all of the large peaks and most of the lesser peaks were identified. At 1000 exposures, all large and small peaks were identified. However, the time trade-off for 1000 exposures was deemed excessive and would only be used for samples where the detail required would offset the time involved.

At 100 exposures, samples with 2-3 significant minerals were readily analyzed and identified. Samples with more than 2-3 significant minerals required a higher number of exposures in order to identify the composition. Samples with a more varied mineral content were run using 250 exposures.

Another variable in the trials was the displayed value of Energy in keV. The basic setup of the InXitu Terra displayed values on the XRF plot from 3 to $10 \mathrm{keV}$. In this range, the typical minerals displayed were calcium, titanium, vanadium, manganese, iron, cobalt, and copper. The cobalt displayed was from the excitation source of the device and was ignored. In order to display other elements of interest, the $\mathrm{keV}$ range was widened to 1 to 15. This allowed magnesium, aluminum, silicon, and arsenic to be displayed. The XRF plot was used to assist in the identification of the minerals later using Jade software.

\section{$\underline{\text { InXitu Terra Software Operation }}$}

The InXitu Terra is pre-programmed to perform the analysis on each sample. By applying the changes detailed in the previous section, the device was set to run automatically. Each sample analysis was completed in approximately 60 minutes. During the analysis, the XRD and XRF plots can be viewed to see patterns developing.

A number of output files are generated with each analysis. The files that were saved for each sample were the XRF plot and the MDI-ka file. The MDI-ka file contained the XRD scan which was used later in the Jade software to identify the minerals present in the sample.

\section{Analyzing the InXitu Terra Output}

Upon completion of the XRD/XRF analysis, the MDI file was read into the Jade software and a Search/Match was performed to identify the peaks in the XRD plot. The Jade software normally identified the top 2-3 minerals present in the sample without intervention. Occasionally, multiples re-runs of the Search/Match function were needed to determine the primary minerals present. 


\section{Appendix 3: SEM Sample Preparation and Imaging Procedure}

The following procedure is used within the WVU Department of Chemical Engineering for imaging samples in the Scanning Electron Microscope (SEM). The device used for imaging sediment samples is the Hitachi S-4700 Field Emission Scanning Electron Microscope. Details of the device are:

Manufacturer: Hitachi

Model: S-4700

Type: Cold Field Emission

\section{Technical characteristics:}

- accelerating voltage: $0.5-30 \mathrm{kV}$

- magnification: X30 - X500,000

- high resolution at low accelerating voltage;

- automatic image processing and data recording

- chemical analysis through an EDS (Energy Dispersive Spectrometer) system

The Hitachi S-4700 SEM operates using a cold field emission electron source. A single crystal tungsten tip with a $100 \mathrm{~nm}$ curvature radius acts as a cathode. Together with two sets of anodes and in a high vacuum environment (as low as 1x10 -8 Pa at the last ion pump), it is able to generate an electron beam with a virtual source size as small as $3 \mathrm{~nm}$ in diameter. The S-4700 combines secondary (SE) and backscattered (BSE) electron detection in a single unit, as well as input from upper and lower detectors.

Elemental analysis (including "light elements" from Boron up) is possible using the energy dispersive spectrometer.

\section{SEM sample preparation and imaging:}

Samples are placed face-up on two-sided carbon tape adhered to an aluminum sample stub.

The samples are then placed in the evacuation/coating chamber of an SPI-module Sputter coater and the chamber is evacuated to less than 4 millitorr. Once this level of vacuum is achieved, the chamber is briefly flushed with argon and restored to a vacuum of less than 2 millitorr.

The argon flow is then restarted and adjusted until the test value of the plasma current is set at 18 milliamps. The timer is then checked to verify 20 seconds of coating time, which under ideal conditions will deposit approximately 60 angstroms of gold-palladium onto the sample, and the timer is started. 
Once the timer has reached zero the plasma discharge is stopped, and the pumps and the Sputter unit are switched off. The evacuation/coating chamber is then returned to atmospheric pressure, and the sample is removed.

Once coated, the sample stub is mounted to the appropriate holder and placed in the Sample Exchange Chamber (SEC) of the SEM (see specifications below for model details). Once vacuum in the SEC is below $4 \times 10^{-3} \mathrm{~Pa}$, the sample assembly is transferred into the Sample Chamber and the High Voltage is applied to the SEM tip. Samples in this series were examined using a $5 \mathrm{kV}$ olt beam, at a working distance of approximately 12 $\mathrm{mm}$, using the lower detector. 


\section{Appendix 4: Lithostatic Force Calculation of Sediment Packing}

To determine the weight used to compact sediment to the same depth as the Mount Elbert saturation deflection, Newton's Second Law was applied.

$$
\Sigma \text { Force }=\Sigma(\text { Mass } \cdot \text { Acceleration }) \text {, or } \Sigma F=\Sigma(\mathrm{m} \cdot \mathrm{a})
$$

In order to equate the lithostatic force experienced at the base of a sediment column to rotational force experienced by a sample in a centrifuge, two forces had to be equated.

$$
\mathrm{F}_{\text {lithostatic }}=\mathrm{F}_{\text {rotational }}
$$

Each force term had to be solved using different variables. The mass term in the lithostatic force was determined by:

$$
\mathrm{m}=\rho \cdot \mathrm{h} \cdot \mathrm{A},
$$

where $\mathrm{m}=$ mass of sediment, $\rho=$ density of sediment, $\mathrm{h}=$ height of sediment, and $\mathrm{A}=$ Area of sediment core.

The acceleration term was earth's gravity $(\mathrm{g})$. The final form of the lithostatic force equation was:

$$
\mathrm{F}_{\text {lithostatic }}=\rho \cdot \mathrm{h} \cdot \mathrm{A} \cdot \mathrm{g}
$$

In order to equate this force to a rotating weight in a centrifuge, Newton's Second Law was recalculated. The mass of the rotating compacting weight was to be calculated. The acceleration term was determined by using the radius of the rotating weight in the centrifuge and the angular velocity. The angular velocity was number of rotations per time. Thus, the acceleration term became:

$$
\text { Acceleration }=\text { radius } \cdot(\text { angular velocity })^{2}, \text { or } \mathrm{a}=\mathrm{r} \cdot \omega^{2}
$$

The rotational force term therefore became:

$$
\mathrm{F}_{\text {rotational }}=\text { mass of compacting weight } \cdot r \cdot \omega^{2}
$$

Equation the two force terms yields the final equation:

$$
\rho \cdot \mathrm{h} \cdot \mathrm{A} \cdot \mathrm{g}=\text { mass of compacting weight } \cdot \mathrm{r} \cdot \omega^{2}
$$

Solving for the mass of the compacting weight:

$$
\text { Mass of compacting weight }=\frac{\rho \cdot h \cdot A \cdot g}{\mathrm{r} \cdot \omega^{2}}
$$

Sediment physical data from Rose, et al $(2011)^{2}$ was used to calculate the dimensions of a stainless steel rod. 


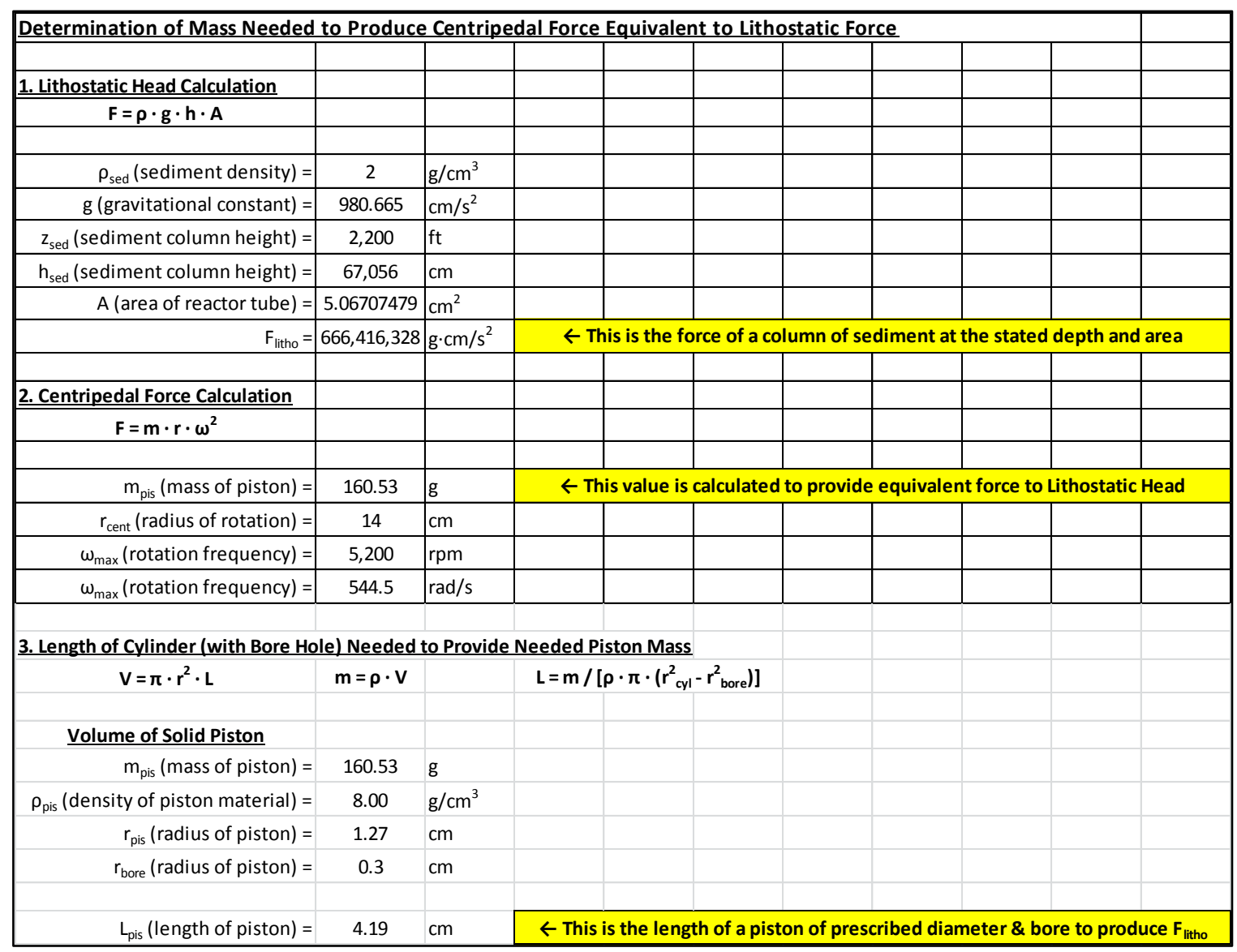

